\title{
Analysis of $\boldsymbol{P}_{\boldsymbol{c}}(\mathbf{4 3 8 0})$ and $\boldsymbol{P}_{\boldsymbol{c}}(\mathbf{4 4 5 0 )}$ as pentaquark states in the diquark model with QCD sum rules
}

\author{
Zhi-Gang Wang ${ }^{\mathrm{a}}$ \\ Department of Physics, North China Electric Power University, Baoding 071003, People's Republic of China
}

Received: 21 December 2015 / Accepted: 1 February 2016 / Published online: 12 February 2016

(C) The Author(s) 2016. This article is published with open access at Springerlink.com

\begin{abstract}
In this article, we construct the diquark-diquarkantiquark type interpolating currents, and we study the masses and pole residues of the $J^{P}=\frac{3}{2}^{-}$and $\frac{5}{2}^{+}$hidden charm pentaquark states in detail with the QCD sum rules by calculating the contributions of the vacuum condensates up to dimension-10 in the operator product expansion. In the calculations, we use the formula $\mu=\sqrt{M_{P_{c}}^{2}-\left(2 \mathbb{M}_{c}\right)^{2}}$ to determine the energy scales of the QCD spectral densities. The present predictions favor assigning $P_{c}(4380)$ and $P_{c}(4450)$ to be the $\frac{3}{2}^{-}$and $\frac{5}{2}^{+}$pentaquark states, respectively.
\end{abstract}

\section{Introduction}

In 1964, Gell-Mann suggested that multiquark states beyond the minimal quark contents $q \bar{q}$ and $q q q$ maybe exist [1]; a quantitative model for the tetraquark states with the quark contents $q q \bar{q} \bar{q}$ was developed by Jaffe using the MIT bag model in 1976 [2]. Later, the five-quark baryons with the quark contents $q q q q \bar{q}$ were developed [3], while the name pentaquark was introduced by Lipkin [4]. QCD allows the existence of multiquark states and hybrid states which contain not only quarks but also gluonic degrees of freedom. We can construct the tetraquark states and pentaquark states according to the diquark-antidiquark model and diquarkdiquark-antiquark model, respectively [5-7]. In the light quark sector, the nature of the scalar mesons below $1 \mathrm{GeV}$ is under controversy [8], although those light tetraquark states are not ruled out in the $N_{c}$ limit [9]. In the heavy quark sector, several $X, Y$, and $Z$ mesons are observed, such as $Z_{c}(3900)^{ \pm}$, $Z_{c}(4020 / 4025)^{ \pm}, Z(4430)^{ \pm}$, the net charge indicates that their constituents are $c \bar{c} u \bar{d}$ or $c \bar{c} d \bar{u}$; for a recent review of both the experimental and the theoretical aspects, one may consult Ref. [10]. Some $X, Y$, and $Z$ mesons are assigned tentatively to be tetraquark states, irrespective of the diquark-

\footnotetext{
a e-mail: zgwang@aliyun.com
}

antidiquark type or the meson-meson type. The two heavy quarks play an important role in stabilizing the multiquark systems, just as in the case of the $\left(\mu^{-} e^{+}\right)\left(\mu^{+} e^{-}\right)$molecule in QED [11]. The spatial separation between the diquark and antidiquark in the tetraquark states [11,12] (or meson and meson in the molecular states [13-15]) may lead to small decay widths. We can study the decay patterns by performing the Fierz re-arrangements non-relativistically in the Paulispinor space [12-15] or relativistically in the Dirac-spinor space [16-19].

Recently, the LHCb collaboration observed two exotic structures $\left(P_{c}(4380)\right.$ and $\left.P_{c}(4450)\right)$ in the $J / \psi p$ mass spectrum in the $\Lambda_{b}^{0} \rightarrow J / \psi K^{-} p$ decays, which are referred to as charmonium-pentaquark states now [20]. $P_{c}(4380)$ has a mass of $4380 \pm 8 \pm 29 \mathrm{MeV}$ and a width of $205 \pm 18 \pm 86 \mathrm{MeV}$, while $P_{c}(4450)$ has a mass of $4449.8 \pm 1.7 \pm 2.5 \mathrm{MeV}$ and a width of $39 \pm 5 \pm 19 \mathrm{MeV}$. The preferred spin-parity assignments of $P_{c}(4380)$ and $P_{c}(4450)$ are $J^{P}=\frac{3}{2}^{-}$and $\frac{5}{2}^{+}$, respectively. The significance of each of the two resonances is more than $9 \sigma$ [20]. The $P_{c}(4380)$ and $P_{c}(4450)$ have attracted much attention of theoretical physicists, and several attempted assignments are suggested, such as $\Sigma_{c} \bar{D}^{*}, \Sigma_{c}^{*} \bar{D}^{*}$, $\chi_{c 1} p$ molecular pentaquark states [21-25] (or not the molecular pentaquark states [26]), the diquark-diquark-antiquark type pentaquark states [27-29], the diquark-triquark type pentaquark states [30], re-scattering effects [31-33], etc. We can test their resonant nature by using photoproduction off a proton target [34-36].

The quarks have color $S U(3)$ symmetry, we can construct the pentaquark states according to the routine quark $\rightarrow$ diquark $\rightarrow$ pentaquark,

$$
\begin{aligned}
(3 \otimes 3) \otimes(3 \otimes 3) \otimes \overline{3} & =(\overline{3} \oplus 6) \otimes(\overline{3} \oplus 6) \otimes \overline{3} \\
& =\overline{3} \otimes \overline{3} \otimes \overline{3} \oplus \cdots=1 \oplus \cdots,
\end{aligned}
$$

or we construct the molecular pentaquark states according to the routine quark $\rightarrow$ meson and baryon $\rightarrow$ molecular 
pentaquark state,

$$
\begin{aligned}
(3 \otimes \overline{3}) \otimes(3 \otimes 3 \otimes 3) & =(1 \oplus 8) \otimes(1 \oplus \cdots) \\
& =(1 \otimes 1) \oplus \cdots=1 \oplus \cdots,
\end{aligned}
$$

where the $1,3(\overline{3}), 6$, and 8 denote the color singlet, triplet (antitriplet), sextet, and octet, respectively. In the diquark model, the pentaquark states consist of two diquarks and an antiquark, which are colored constituents; it is easy to form compact bound states due to the strong attractions at long distance. In the meson-baryon model, the molecular pentaquark states consist of a colorless meson and a colorless baryon, and attractions induced by exchanges of the intermediate mesons (Yukawa-like potentials) are needed to form loose bound states. In this article, we take the $P_{c}$ (4380) and $P_{c}(4450)$ as the diquark-diquark-antiquark type pentaquark states, construct the interpolating currents consist of five quarks according to Eq. (1), and study their masses and pole residues with the QCD sum rules.

In previous work, we described the hidden charm (or bottom) four-quark systems $q \bar{q}^{\prime} Q \bar{Q}$ by a double-well potential [16-19,37-39]. In the four-quark system $q \bar{q}^{\prime} Q \bar{Q}$, the $Q$ quark serves as a static well potential and combines with the light quark $q$ to form a heavy diquark $\mathcal{D}_{q Q}^{i}$ in a color antitriplet [16-19],

$q+Q \rightarrow \mathcal{D}_{q Q}^{i}$,

or it combines with the light antiquark $\bar{q}^{\prime}$ to form a heavy meson in a color singlet (meson-like state in a color octet) [37-39],

$\bar{q}^{\prime}+Q \rightarrow \bar{q}^{\prime} Q\left(\bar{q}^{\prime} \lambda^{a} Q\right)$,

the $\bar{Q}$-quark serves as another static well potential and combines with the light antiquark $\bar{q}^{\prime}$ to form a heavy antidiquark $\mathcal{D}_{\bar{q}^{\prime} \bar{Q}}^{i}$ in a color triplet [16-19],

$\bar{q}^{\prime}+\bar{Q} \rightarrow \mathcal{D}_{\bar{q}^{\prime} \bar{Q}}^{i}$,

or it combines with the light quark $q$ to form a heavy meson in a color singlet (meson-like state in a color octet) [37-39],

$q+\bar{Q} \rightarrow \bar{Q} q\left(\bar{Q} \lambda^{a} q\right)$

where $i$ is the color index and $\lambda^{a}$ is a Gell-Mann matrix. Then

$$
\begin{aligned}
\mathcal{D}_{q Q}^{i}+\mathcal{D}_{\bar{q}^{\prime} \bar{Q}}^{i} & \rightarrow \text { compact tetraquark states } \\
\bar{q}^{\prime} Q+\bar{Q} q & \rightarrow \text { loose molecular states } \\
\bar{q}^{\prime} \lambda^{a} Q+\bar{Q} \lambda^{a} q & \rightarrow \text { molecule-like states }
\end{aligned}
$$

the two heavy quarks $Q$ and $\bar{Q}$ stabilize the four-quark systems $q \bar{q}^{\prime} Q \bar{Q}$, just as in the case of the $\left(\mu^{-} e^{+}\right)\left(\mu^{+} e^{-}\right)$ molecule in QED [11].

The hidden charm (or bottom) five-quark systems $q q_{1} q_{2} Q$ $\bar{Q}$ can also be described by a double-well potential by using the replacement

$q_{1}+q_{2}+\bar{Q} \rightarrow \mathcal{D}_{q_{1} q_{2}\left(\bar{q}^{\prime}\right)}^{j}+\bar{Q}^{k} \rightarrow \mathcal{T}_{q_{1} q_{2}\left(\bar{q}^{\prime}\right) \bar{Q}}^{i}$,

just like the four-quark systems $q \bar{q}^{\prime} Q \bar{Q}[16-19,37,38]$, where $\mathcal{T}_{q_{1} q_{2}}^{i} \bar{Q}$ denotes the heavy triquark in a color triplet, the $\bar{q}^{\prime}$ in the bracket denotes that $\mathcal{D}_{q_{1} q_{2}}^{j}$ is in a color antitriplet, just like the $\bar{q}^{\prime j}$. In the heavy quark limit, the $Q$-quark $(\bar{Q}$ quark) can be taken as a static well potential, the diquark $\mathcal{D}_{q_{1} q_{2}}^{j}$ and quark $q$ lie in the two wells, respectively.

The QCD sum rules have been applied extensively to study the hidden charm (bottom) tetraquark states [40-45], however, the energy-scale dependence of the QCD spectral densities is not studied. In previous work, we studied the acceptable energy scales of the QCD spectral densities for the hidden charm (bottom) tetraquark states and molecular (and molecule-like) states in the QCD sum rules in detail for the first time [16-19,37-39,46-48], and suggested the formula

$\mu=\sqrt{M_{X / Y / Z}^{2}-\left(2 \mathbb{M}_{Q}\right)^{2}}$

to determine the energy scales based on the analysis in Eqs. (3), (4), (5), (6), and (7), where $X, Y$, and $Z$ denote the fourquark systems, and the $\mathbb{M}_{Q}$ denotes the effective heavy quark masses [16-19,37-39]. The energy-scale formula works well for all the tetraquark states, molecular states, and moleculelike states.

In the non-relativistic quark model, the heavy quarks have finite masses, which quantitatively affect the spin-spin interactions between the quarks within one diquark or in two different diquarks [12]. In the QCD sum rules, the net effects of the different dynamics are embodied in the effective masses $\mathbb{M}_{c}$ and $\mathbb{M}_{b}$, respectively; for example, $Z_{c}$ (3900) and $Z_{b}(10610)$ can be tentatively assigned to be the $J^{P C}=$ $1^{+-}$tetraquark states with the symbolic quark structures $\frac{[c u]_{S=0}[\bar{c} \bar{d}]_{S=1}-[c u]_{S=1}[\bar{c} \bar{d}]_{S=0}}{\sqrt{2}}$ and $\frac{[b u]_{S=0}[\bar{b} \bar{d}]_{S=1}-[b u]_{S=1}[\bar{b} \bar{d}]_{S=0}}{\sqrt{2}}$, respectively, where the subscript $S$ denotes the spin. The optimal energy scales of their QCD spectral densities are quite different, $\mu_{Z_{c}(3900)}=1.5 \mathrm{GeV}$ and $\mu_{Z_{b}(10610)}=2.7 \mathrm{GeV}$ $[16-19,46]$, although they are cousins. Meanwhile, in the heavy quark limit $m_{Q} \rightarrow \infty$, we naively expect that the two energy scales $\mu_{Z_{c}(3900)}$ and $\mu_{Z_{b}(10610)}$ coincide. In this work, we extend the energy-scale formula to study the diquarkdiquark-antiquark type pentaquark states, and we try to assign $P_{c}(4380)$ and $P_{c}(4450)$ to be the $\frac{3}{2}^{-}$and $\frac{5}{2}^{+}$pentaquark states, respectively.

The article is arranged as follows: we derive the QCD sum rules for the masses and pole residues of $P_{c}$ (4380) and $P_{c}(4450)$ in Sect.2; in Sect.3, we present the numerical results and discussions; Sect. 4 is reserved for our conclusions. 


\section{QCD sum rules for $P_{c}(4380)$ and $P_{c}(4450)$}

In the following, we write down the two-point correlation functions $\Pi_{\mu \nu}(p)$ and $\Pi_{\mu \nu \alpha \beta}(p)$ in the QCD sum rules,

$$
\begin{gathered}
\Pi_{\mu \nu}(p)=i \int \mathrm{d}^{4} x e^{i p \cdot x}\left\langle 0\left|T\left\{J_{\mu}(x) \bar{J}_{\nu}(0)\right\}\right| 0\right\rangle, \\
\Pi_{\mu \nu \alpha \beta}(p)=i \int \mathrm{d}^{4} x e^{i p \cdot x}\left\langle 0\left|T\left\{J_{\mu \nu}(x) \bar{J}_{\alpha \beta}(0)\right\}\right| 0\right\rangle,
\end{gathered}
$$

where

$$
\begin{aligned}
J_{\mu}(x)= & \varepsilon^{i l a} \varepsilon^{i j k} \varepsilon^{l m n} u_{j}^{T}(x) C \gamma_{5} d_{k}(x) u_{m}^{T}(x) \\
& \times C \gamma_{\mu} c_{n}(x) C \bar{c}_{a}^{T}(x), \\
J_{\mu \nu}(x)= & \frac{1}{\sqrt{2}} \varepsilon^{i l a} \varepsilon^{i j k} \varepsilon^{l m n} u_{j}^{T}(x) C \gamma_{5} d_{k}(x) \\
& \times\left[u_{m}^{T}(x) C \gamma_{\mu} c_{n}(x) \gamma_{\nu} C \bar{c}_{a}^{T}(x)+u_{m}^{T}(x)\right. \\
& \left.\times C \gamma_{\nu} c_{n}(x) \gamma_{\mu} C \bar{c}_{a}^{T}(x)\right],
\end{aligned}
$$

$i, j, k, \ldots$ are color indices, and $C$ is the charge conjugation matrix. The diquarks $q_{j}^{T} C \Gamma q_{k}^{\prime}$ have five structures in Diracspinor space, where $C \Gamma=C \gamma_{5}, C, C \gamma_{\mu} \gamma_{5}, C \gamma_{\mu}$, and $C \sigma_{\mu \nu}$ for the scalar, pseudoscalar, vector, axial-vector, and tensor diquarks, respectively. The structures $C \gamma_{\mu}$ and $C \sigma_{\mu \nu}$ are symmetric, while the structures $C \gamma_{5}, C$, and $C \gamma_{\mu} \gamma_{5}$ are antisymmetric. The scattering amplitude for one-gluon exchange is proportional to

$$
\begin{aligned}
\left(\frac{\lambda^{a}}{2}\right)_{k i}\left(\frac{\lambda^{a}}{2}\right)_{l j}= & -\frac{1}{3}\left(\delta_{j k} \delta_{i l}-\delta_{i k} \delta_{j l}\right) \\
& +\frac{1}{6}\left(\delta_{j k} \delta_{i l}+\delta_{i k} \delta_{j l}\right),
\end{aligned}
$$

where $i, j$ and $k, l$ are the color indices of the two quarks in the incoming and outgoing channels, respectively. The negative sign in front of the antisymmetric antitriplet indicates that the interaction is attractive, while the positive sign in front of the symmetric sextet indicates the interaction is repulsive. The attractive interactions of one-gluon exchange favor the formation of the diquarks in a color antitriplet $\overline{3}_{c}$, flavor antitriplet $\overline{3}_{f}$, and spin singlet $1_{s}[49,50]$, while the favored configurations are the scalar $\left(C \gamma_{5}\right)$ and axial-vector $\left(C \gamma_{\mu}\right)$ diquark states [51-53]. The calculations based on the QCD sum rules indicate that the heavy-light scalar and axialvector diquark states have almost degenerate masses [51,52], while the masses of the light axial-vector diquark states lie (150-200) $\mathrm{MeV}$ above that of the light scalar diquark states [53], if they have the same quark constituents. In this article, we choose the light scalar diquark and heavy axialvector diquark as basic constituents, and construct the scalar diquark-axial-vector diquark-antiquark type currents $J_{\mu}(x)$ and $J_{\mu \nu}$ with the spin-parity $\frac{3}{2}^{-}$and $\frac{5}{2}^{+}$, respectively, to interpolate the pentaquark states $P_{c}(4380)$ and $P_{c}(4450)$, respectively; see Eqs. (3) and (8).
In fact, we can also construct the axial-vector diquarkscalar diquark-antiquark type current $\eta_{\mu}(x)$ and the axialvector diquark-axial-vector diquark-antiquark type current $\eta_{\mu \nu}(x)$,

$$
\begin{aligned}
\eta_{\mu}(x)= & \frac{\varepsilon^{i l a} \varepsilon^{i j k} \varepsilon^{l m n}}{\sqrt{3}}\left[u_{j}^{T}(x) C \gamma_{\mu} u_{k}(x) d_{m}^{T}(x) C \gamma_{5} c_{n}(x)\right. \\
& \left.+2 u_{j}^{T}(x) C \gamma_{\mu} d_{k}(x) u_{m}^{T}(x) C \gamma_{5} c_{n}(x)\right] C \bar{c}_{a}^{T}(x), \\
\eta_{\mu \nu}(x)= & \frac{\varepsilon^{i l a} \varepsilon^{i j k} \varepsilon^{l m n}}{\sqrt{6}}\left[u_{j}^{T}(x) C \gamma_{\mu} u_{k}(x) d_{m}^{T}(x) C \gamma_{\nu} c_{n}(x)\right. \\
& \left.+2 u_{j}^{T}(x) C \gamma_{\mu} d_{k}(x) u_{m}^{T}(x) C \gamma_{\nu} c_{n}(x)\right] \gamma_{5} C \bar{c}_{a}^{T}(x) \\
& +(\mu \leftrightarrow \nu),
\end{aligned}
$$

to study the spin-parity $\frac{3}{2}^{-}$and $\frac{5}{2}^{+}$pentaquark states, respectively. As the masses of the light axial-vector diquark states lie (150-200) MeV above that of the corresponding light scalar diquark states [53]. The currents $\eta_{\mu}(x)$ and $\eta_{\mu \nu}(x)$ are supposed to couple to the pentaquark states with larger masses compared to the currents $J_{\mu}(x)$ and $J_{\mu \nu}(x)$, respectively.

The $\Lambda_{b}^{0}$ can be well interpolated by the current $J(x)=$ $\varepsilon^{i j k} u_{i}^{T}(x) C \gamma_{5} d_{j}(x) b_{k}(x)$ [54], the $u$ and $d$ quarks in the $\Lambda_{b}^{0}$ form a scalar diquark $[u d]$ in color antitriplet, the decays $\Lambda_{b}^{0} \rightarrow J / \psi p K^{-}$take place through the mechanism,

$$
\begin{aligned}
\Lambda_{b}^{0}([u d] b) & \rightarrow[u d] c \bar{c} s \rightarrow[u d] c \bar{c} u \bar{u} s \\
& \rightarrow P_{c}^{+}([u d][u c] \bar{c}) K^{-}(\bar{u} s) \rightarrow J / \psi p K^{-},
\end{aligned}
$$

at the quark level. In the decays $P_{c}^{+}([u d][u c] \bar{c}) \rightarrow J / \psi p$, the scalar diquark $[u d]$ survives in the decays, the decays are greatly facilitated. On the other hand, if there exists a light axial-vector diquark $[u d]$, which has to dissolve to form a scalar diquark $[u d]$, the decays are not facilitated.

The currents $J_{\mu}(0)$ and $J_{\mu \nu}(0)$ couple potentially to $\frac{1}{2}^{+}$, $\frac{3}{2}^{-}$, and $\frac{1}{2}^{+}, \frac{3}{2}^{-}, \frac{5}{2}^{+}$hidden charm pentaquark states $P_{\frac{1}{2}}^{+}$, $P_{\frac{3}{2}}^{-}$, and $P_{\frac{1}{2}}^{+}, P_{\frac{3}{2}}^{-}, P_{\frac{5}{2}}^{+}$, respectively,

$\left\langle 0\left|J_{\mu}(0)\right| P_{\frac{1}{2}}^{+}(p)\right\rangle=f_{\frac{1}{2}}^{+} p_{\mu} U^{+}(p, s)$,

$\left\langle 0\left|J_{\mu}(0)\right| P_{\frac{3}{2}}^{-}(p)\right\rangle=\lambda_{\frac{3}{2}}^{-} U_{\mu}^{-}(p, s)$,

$\left\langle 0\left|J_{\mu \nu}(0)\right| P_{\frac{1}{2}}^{+}(p)\right\rangle=g_{\frac{1}{2}}^{+} p_{\mu} p_{\nu} U^{+}(p, s)$,

$\left\langle 0\left|J_{\mu \nu}(0)\right| P_{\frac{3}{2}}^{-}(p)\right\rangle=f_{\frac{3}{2}}^{-}\left[p_{\mu} U_{\nu}^{-}(p, s)+p_{\nu} U_{\mu}^{-}(p, s)\right]$,

$\left\langle 0\left|J_{\mu \nu}(0)\right| P_{\frac{5}{2}}^{+}(p)\right\rangle=\lambda_{\frac{5}{2}}^{+} U_{\mu \nu}^{+}(p, s)$,

the spinors $U^{ \pm}(p, s)$ satisfy the Dirac equations $(/ p-$ $\left.M_{ \pm}\right) U^{ \pm}(p)=0$, while the spinors $U_{\mu}^{ \pm}(p, s)$ and $U_{\mu \nu}^{ \pm}(p, s)$ 
satisfy the Rarita-Schwinger equations $\left(\not p-M_{ \pm}\right) U_{\mu}^{ \pm}(p)=0$ and $\left(\not p-M_{ \pm}\right) U_{\mu \nu}^{ \pm}(p)=0$, and the relations $\gamma^{\mu} U_{\mu}^{ \pm}(p, s)=$ $0, p^{\mu} U_{\mu}^{ \pm}(p, s)=0, \gamma^{\mu} U_{\mu \nu}^{ \pm}(p, s)=0, p^{\mu} U_{\mu \nu}^{ \pm}(p, s)=0$, and $U_{\mu \nu}^{ \pm}(p, s)=U_{\nu \mu}^{ \pm}(p, s)$, respectively. On the other hand, the currents $J_{\mu}(0)$ and $J_{\mu \nu}(0)$ also couple potentially to the $\frac{1}{2}^{-}, \frac{3}{2}^{+}$, and $\frac{1}{2}^{-}, \frac{3}{2}^{+}, \frac{5}{2}^{-}$hidden charm pentaquark states $P_{\frac{1}{2}}^{-}, P_{\frac{3}{2}}^{+}, P_{\frac{1}{2}}^{-}, P_{\frac{3}{2}}^{+}$, and $P_{\frac{5}{2}}^{-}$, respectively,

$\left\langle 0\left|J_{\mu}(0)\right| P_{\frac{1}{2}}^{-}(p)\right\rangle=f_{\frac{1}{2}}^{-} p_{\mu} i \gamma_{5} U^{-}(p, s)$,

$\left\langle 0\left|J_{\mu}(0)\right| P_{\frac{3}{2}}^{+}(p)\right\rangle=\lambda_{\frac{3}{2}}^{+} i \gamma_{5} U_{\mu}^{+}(p, s)$,

$\left\langle 0\left|J_{\mu \nu}(0)\right| P_{\frac{1}{2}}^{-}(p)\right\rangle=g_{\frac{1}{2}}^{-} p_{\mu} p_{\nu} i \gamma_{5} U^{-}(p, s)$,

$\left\langle 0\left|J_{\mu \nu}(0)\right| P_{\frac{3}{2}}^{+}(p)\right\rangle=f_{\frac{3}{2}}^{+} i \gamma_{5}\left[p_{\mu} U_{\nu}^{+}(p, s)+p_{\nu} U_{\mu}^{+}(p, s)\right]$,

$\left\langle 0\left|J_{\mu \nu}(0)\right| P_{\frac{5}{2}}^{-}(p)\right\rangle=\lambda_{\frac{5}{2}}^{-} i \gamma_{5} U_{\mu \nu}^{-}(p, s)$,

the spinors $U_{\mu}^{-}(p, s)$ and $U_{\mu}^{+}(p, s) \quad\left(U_{\mu \nu}^{-}(p, s)\right.$ and $\left.U_{\mu \nu}^{+}(p, s)\right)$ have analogous properties, and the pole residues obey $\lambda_{\frac{3}{2} / \frac{5}{2}}^{ \pm} \neq 0, f_{\frac{1}{2} / \frac{3}{2}}^{ \pm} \neq 0$, and $g_{\frac{1}{2}}^{ \pm} \neq 0$.

We insert a complete set of intermediate pentaquark states with the same quantum numbers as the current operators $J_{\mu}(x), i \gamma_{5} J_{\mu}(x), J_{\mu \nu}(x)$, and $i \gamma_{5} J_{\mu \nu}(x)$ into the correlation functions $\Pi_{\mu \nu}(p)$ and $\Pi_{\mu \nu \alpha \beta}(p)$ to obtain the hadronic representation $[55,56]$. After isolating the pole terms of the lowest states of the hidden charm pentaquark states, we obtain the following results:

$$
\begin{aligned}
\Pi_{\mu \nu}(p)= & \lambda_{\frac{3}{2}}^{-2} \frac{p p+M_{-}}{M_{-}^{2}-p^{2}}\left(-g_{\mu \nu}+\frac{\gamma_{\mu} \gamma_{\nu}}{3}+\frac{2 p_{\mu} p_{v}}{3 p^{2}}\right. \\
& \left.-\frac{p_{\mu} \gamma_{\nu}-p_{\nu} \gamma_{\mu}}{3 \sqrt{p^{2}}}\right) \\
& +\lambda_{\frac{3}{2}}^{+2} \frac{\not p-M_{+}}{M_{+}^{2}-p^{2}}\left(-g_{\mu \nu}+\frac{\gamma_{\mu} \gamma_{\nu}}{3}+\frac{2 p_{\mu} p_{v}}{3 p^{2}}\right. \\
& \left.-\frac{p_{\mu} \gamma_{\nu}-p_{\nu} \gamma_{\mu}}{3 \sqrt{p^{2}}}\right) \\
& +f_{\frac{1}{2}}^{+2} \frac{\not p+M_{+}}{M_{+}^{2}-p^{2}} p_{\mu} p_{v}+f_{\frac{1}{2}}^{-2} \frac{\not p-M_{-}}{M_{-}^{2}-p^{2}} p_{\mu} p_{v}+\cdots,
\end{aligned}
$$

$$
\begin{aligned}
\Pi_{\mu \nu \alpha \beta}(p)= & \lambda_{\frac{5}{2}}^{+2} \frac{p p+M_{+}}{M_{+}^{2}-p^{2}}\left[\frac{\widetilde{g}_{\mu \alpha} \widetilde{g}_{\nu \beta}+\widetilde{g}_{\mu \beta} \widetilde{g}_{\nu \alpha}}{2}-\frac{\widetilde{g}_{\mu \nu} \widetilde{g}_{\alpha \beta}}{5}\right. \\
& -\frac{1}{10}\left(\gamma_{\mu} \gamma_{\alpha}+\frac{\gamma_{\mu} p_{\alpha}-\gamma_{\alpha} p_{\mu}}{\sqrt{p^{2}}}-\frac{p_{\mu} p_{\alpha}}{p^{2}}\right) \widetilde{g}_{\nu \beta} \\
& \left.-\frac{1}{10}\left(\gamma_{\nu} \gamma_{\alpha}+\frac{\gamma_{\nu} p_{\alpha}-\gamma_{\alpha} p_{v}}{\sqrt{p^{2}}}-\frac{p_{\nu} p_{\alpha}}{p^{2}}\right) \widetilde{g}_{\mu \beta}+\cdots\right] \\
& +\lambda_{\frac{5}{2}}^{-2} \frac{\not p-M_{-}}{M_{-}^{2}-p^{2}}\left[\frac{\widetilde{g}_{\mu \alpha} \widetilde{g}_{\nu \beta}+\widetilde{g}_{\mu \beta} \widetilde{g}_{\nu \alpha}}{2}-\frac{\widetilde{g}_{\mu \nu} \widetilde{g}_{\alpha \beta}}{5}\right.
\end{aligned}
$$

$$
\begin{aligned}
& -\frac{1}{10}\left(\gamma_{\mu} \gamma_{\alpha}+\frac{\gamma_{\mu} p_{\alpha}-\gamma_{\alpha} p_{\mu}}{\sqrt{p^{2}}}-\frac{p_{\mu} p_{\alpha}}{p^{2}}\right) \tilde{g}_{\nu \beta} \\
& \left.-\frac{1}{10}\left(\gamma_{\nu} \gamma_{\alpha}+\frac{\gamma_{\nu} p_{\alpha}-\gamma_{\alpha} p_{v}}{\sqrt{p^{2}}}-\frac{p_{\nu} p_{\alpha}}{p^{2}}\right) \tilde{g}_{\mu \beta}+\cdots\right] \\
& +f_{\frac{3}{2}}^{-2} \frac{\not p+M_{-}}{M_{-}^{2}-p^{2}}\left[p _ { \mu } p _ { \alpha } \left(-g_{\nu \beta}+\frac{\gamma_{\nu} \gamma_{\beta}}{3}\right.\right. \\
& \left.\left.+\frac{2 p_{v} p_{\beta}}{3 p^{2}}-\frac{p_{\nu} \gamma_{\beta}-p_{\beta} \gamma_{v}}{3 \sqrt{p^{2}}}\right)+\cdots\right] \\
& +f_{\frac{3}{2}}^{+2} \frac{p p-M_{+}}{M_{+}^{2}-p^{2}}\left[p _ { \mu } p _ { \alpha } \left(-g_{\nu \beta}+\frac{\gamma_{\nu} \gamma_{\beta}}{3}\right.\right. \\
& \left.\left.+\frac{2 p_{v} p_{\beta}}{3 p^{2}}-\frac{p_{v} \gamma_{\beta}-p_{\beta} \gamma_{v}}{3 \sqrt{p^{2}}}\right)+\cdots\right] \\
& +g_{\frac{1}{2}}^{+2} \frac{\not p+M_{+}}{M_{+}^{2}-p^{2}} p_{\mu} p_{\nu} p_{\alpha} p_{\beta} \\
& +g_{\frac{1}{2}}^{-2} \frac{\not p-M_{-}}{M_{-}^{2}-p^{2}} p_{\mu} p_{v} p_{\alpha} p_{\beta}+\cdots,
\end{aligned}
$$

where $\widetilde{g}_{\mu \nu}=g_{\mu \nu}-\frac{p_{\mu} p_{v}}{p^{2}}$, the $M_{ \pm}$are the masses of the lowest pentaquark states with the parity \pm , respectively, and $\lambda_{\frac{3}{2} / \frac{5}{2}}^{ \pm}$, $f_{\frac{1}{2} / \frac{3}{2}}^{ \pm}$, and $g_{\frac{1}{2}}^{ \pm}$are the corresponding pole residues. In the calculations, we have used the following summations [57]:

$$
\begin{aligned}
& \sum_{s} U_{\mu} \bar{U}_{\nu}=\left(\not p+M_{ \pm}\right) \\
& \times\left(-g_{\mu \nu}+\frac{\gamma_{\mu} \gamma_{\nu}}{3}+\frac{2 p_{\mu} p_{\nu}}{3 p^{2}}-\frac{p_{\mu} \gamma_{\nu}-p_{\nu} \gamma_{\mu}}{3 \sqrt{p^{2}}}\right), \\
& \sum_{s} U_{\mu \nu} \bar{U}_{\alpha \beta}=\left(\not p+M_{ \pm}\right)\left\{\frac{\tilde{g}_{\mu \alpha} \tilde{g}_{\nu \beta}+\tilde{g}_{\mu \beta} \tilde{g}_{\nu \alpha}}{2}-\frac{\widetilde{g}_{\mu \nu} \widetilde{g}_{\alpha \beta}}{5}\right. \\
& -\frac{1}{10}\left(\gamma_{\mu} \gamma_{\alpha}+\frac{\gamma_{\mu} p_{\alpha}-\gamma_{\alpha} p_{\mu}}{\sqrt{p^{2}}}-\frac{p_{\mu} p_{\alpha}}{p^{2}}\right) \widetilde{g}_{\nu \beta} \\
& -\frac{1}{10}\left(\gamma_{\nu} \gamma_{\alpha}+\frac{\gamma_{\nu} p_{\alpha}-\gamma_{\alpha} p_{\nu}}{\sqrt{p^{2}}}-\frac{p_{\nu} p_{\alpha}}{p^{2}}\right) \tilde{g}_{\mu \beta} \\
& -\frac{1}{10}\left(\gamma_{\mu} \gamma_{\beta}+\frac{\gamma_{\mu} p_{\beta}-\gamma_{\beta} p_{\mu}}{\sqrt{p^{2}}}-\frac{p_{\mu} p_{\beta}}{p^{2}}\right) \widetilde{g}_{\nu \alpha} \\
& \left.-\frac{1}{10}\left(\gamma_{\nu} \gamma_{\beta}+\frac{\gamma_{\nu} p_{\beta}-\gamma_{\beta} p_{v}}{\sqrt{p^{2}}}-\frac{p_{\nu} p_{\beta}}{p^{2}}\right) \widetilde{g}_{\mu \alpha}\right\},
\end{aligned}
$$

and $p^{2}=M_{ \pm}^{2}$ on the mass-shell.

We can rewrite the correlation functions $\Pi_{\mu \nu}(p)$ and $\Pi_{\mu \nu \alpha \beta}(p)$ into the following form according to Lorentz covariance:

$$
\begin{aligned}
\Pi_{\mu \nu}(p)= & \Pi_{\frac{3}{2}}\left(p^{2}\right)\left(-g_{\mu \nu}\right)+\Pi_{\frac{3}{2}}^{1}\left(p^{2}\right) \gamma_{\mu} \gamma_{\nu} \\
& +\Pi_{\frac{3}{2}}^{2}\left(p^{2}\right)\left(p_{\mu} \gamma_{\nu}-p_{\nu} \gamma_{\mu}\right)+\Pi_{\frac{1}{2}, \frac{3}{2}}\left(p^{2}\right) p_{\mu} p_{\nu},
\end{aligned}
$$




$$
\begin{aligned}
\Pi_{\mu \nu \alpha \beta}(p)= & \Pi_{\frac{5}{2}}\left(p^{2}\right) \frac{g_{\mu \alpha} g_{\nu \beta}+g_{\mu \beta} g_{\nu \alpha}}{2}+\Pi_{\frac{5}{2}}^{1}\left(p^{2}\right) g_{\mu \nu} g_{\alpha \beta} \\
& +\Pi_{\frac{5}{2}}^{2}\left(p^{2}\right)\left(g_{\mu \nu} p_{\alpha} p_{\beta}+g_{\alpha \beta} p_{\mu} p_{\nu}\right) \\
& +\Pi_{\frac{5}{2}}^{3}\left(p^{2}\right)\left(g_{\mu \alpha} \gamma_{\nu} \gamma_{\beta}+g_{\mu \beta} \gamma_{\nu} \gamma_{\alpha}\right. \\
& \left.+g_{\nu \alpha} \gamma_{\mu} \gamma_{\beta}+g_{\nu \beta} \gamma_{\mu} \gamma_{\alpha}\right) \\
& +\Pi_{\frac{5}{2}}^{4}\left(p^{2}\right)\left[g_{\nu \beta}\left(\gamma_{\mu} p_{\alpha}-\gamma_{\alpha} p_{\mu}\right)\right. \\
& +g_{\nu \alpha}\left(\gamma_{\mu} p_{\beta}-\gamma_{\beta} p_{\mu}\right) \\
& \left.+g_{\mu \beta}\left(\gamma_{\nu} p_{\alpha}-\gamma_{\alpha} p_{\nu}\right)+g_{\mu \alpha}\left(\gamma_{\nu} p_{\beta}-\gamma_{\beta} p_{\nu}\right)\right] \\
& +\Pi_{\frac{3}{2}, \frac{5}{2}}^{1}\left(p^{2}\right)\left(g_{\mu \alpha} p_{\nu} p_{\beta}+g_{\mu \beta} p_{\nu} p_{\alpha}\right. \\
& \left.+g_{\nu \alpha} p_{\mu} p_{\beta}+g_{\nu \beta} p_{\mu} p_{\alpha}\right) \\
& +\Pi_{\frac{3}{2}, \frac{5}{2}}^{2}\left(p^{2}\right)\left(\gamma_{\mu} \gamma_{\alpha} p_{\nu} p_{\beta}\right. \\
& \left.+\gamma_{\mu} \gamma_{\beta} p_{\nu} p_{\alpha}+\gamma_{\nu} \gamma_{\alpha} p_{\mu} p_{\beta}+\gamma_{\nu} \gamma_{\beta} p_{\mu} p_{\alpha}\right) \\
& +\Pi_{\frac{3}{2}, \frac{5}{2}}^{3}\left(p^{2}\right)\left[\left(\gamma_{\mu} p_{\alpha}-\gamma_{\alpha} p_{\mu}\right) p_{\nu} p_{\beta}\right. \\
& +\left(\gamma_{\mu} p_{\beta}-\gamma_{\beta} p_{\mu}\right) p_{\nu} p_{\alpha}+\left(\gamma_{\nu} p_{\alpha}-\gamma_{\alpha} p_{\nu}\right) p_{\mu} p_{\beta} \\
& \left.+\left(\gamma_{\nu} p_{\beta}-\gamma_{\beta} p_{\nu}\right) p_{\mu} p_{\alpha}\right] \\
& +\Pi_{\frac{1}{2}, \frac{3}{2}, \frac{5}{2}}\left(p^{2}\right) p_{\mu} p_{\nu} p_{\alpha} p_{\beta},
\end{aligned}
$$

the subscripts $\frac{1}{2}, \frac{3}{2}$, and $\frac{5}{2}$ in the components $\Pi_{\frac{3}{2}}\left(p^{2}\right)$, $\Pi_{\frac{3}{2}}^{1}\left(p^{2}\right), \Pi_{\frac{3}{2}}^{2}\left(p^{2}\right), \Pi_{\frac{1}{2}, \frac{3}{2}}\left(p^{2}\right), \Pi_{\frac{5}{2}}\left(p^{2}\right), \Pi_{\frac{5}{2}}^{1}\left(p^{2}\right), \Pi_{\frac{5}{2}}^{\frac{2}{2}}\left(p^{2}\right)$, $\Pi_{\frac{5}{2}}^{3}\left(p^{2}\right), \Pi_{\frac{5}{2}}^{4}\left(p^{2}\right), \Pi_{\frac{3}{2}, \frac{5}{2}}^{1}\left(p^{2}\right), \Pi_{\frac{3}{2}}^{2}, \frac{5}{2}\left(p^{2}\right), \Pi_{\frac{3}{2}, \frac{5}{2}}^{3}\left(p^{2}\right)$, and $\Pi_{\frac{1}{2}, \frac{3}{2}, \frac{5}{2}}\left(p^{2}\right)$ denote the spins of the pentaquark states, which means that the pentaquark states with $J=\frac{1}{2}, \frac{3}{2}$, and $\frac{5}{2}$ have contributions. The components $\Pi_{\frac{1}{2}, \frac{3}{2}}\left(p^{2}\right), \Pi_{\frac{3}{2}, \frac{5}{2}}^{1}\left(p^{2}\right)$, $\Pi_{\frac{3}{2}, \frac{5}{2}}^{2}\left(p^{2}\right), \Pi_{\frac{3}{2}, \frac{5}{2}}^{3}\left(p^{2}\right)$, and $\Pi_{\frac{1}{2}, \frac{3}{2}, \frac{5}{2}}\left(p^{2}\right)$ receive contributions from more than one pentaquark state, so they can be neglected. We can rewrite $\gamma_{\mu} \gamma_{\nu}=g_{\mu \nu}-i \sigma_{\mu \nu}$; then the components $\Pi_{\frac{3}{2}}^{1}\left(p^{2}\right), \Pi_{\frac{3}{2}}^{2}\left(p^{2}\right), \Pi_{\frac{5}{2}}^{3}\left(p^{2}\right)$, and $\Pi_{\frac{5}{2}}^{4}\left(p^{2}\right)$ are associated with tensor structures which are antisymmetric in the Lorentz indices $\mu, v, \alpha$ or $\beta$. In the calculations, we observe that such antisymmetric properties lead to smaller intervals of dimensions of the vacuum condensates, and therefore worse QCD sum rules, so the components $\Pi_{\frac{3}{2}}^{1}\left(p^{2}\right), \Pi_{\frac{3}{2}}^{2}\left(p^{2}\right)$, $\Pi_{\frac{5}{2}}^{3}\left(p^{2}\right)$, and $\Pi_{\frac{5}{2}}^{4}\left(p^{2}\right)$ can also be neglected. If we take the replacement $J_{\mu \nu}(x) \rightarrow \widehat{J}_{\mu \nu}(x)=J_{\mu \nu}(x)-\frac{1}{4} g_{\mu \nu} J_{\alpha}{ }^{\alpha}(x)$ to subtract the contributions of the $J=\frac{1}{2}$ pentaquark states, a lot of terms $\propto g_{\mu \nu}, g_{\alpha \beta}$ disappear at the QCD side, and this results in smaller intervals of the dimensions of the vacuum condensates, so the components $\Pi_{\frac{5}{2}}^{1}\left(p^{2}\right)$ and $\Pi_{\frac{5}{2}}^{2}\left(p^{2}\right)$ are not the optimal choices to study the $J=\frac{5}{2}$ pentaquark states. Now only the components $\Pi_{\frac{3}{2}}\left(p^{2}\right)$ and $\Pi_{\frac{5}{2}}\left(p^{2}\right)$ are left. The present conclusion is tentative, we can obtain a definite conclusion by obtaining the QCD sum rules based on the components $\Pi_{\frac{3}{2}}^{1}\left(p^{2}\right), \Pi_{\frac{3}{2}}^{2}\left(p^{2}\right), \Pi_{\frac{5}{2}}^{1}\left(p^{2}\right), \Pi_{\frac{5}{2}}^{2}\left(p^{2}\right), \Pi_{\frac{5}{2}}^{3}\left(p^{2}\right)$, and $\Pi_{\frac{5}{2}}^{4}\left(p^{2}\right)$. In this article, we choose the tensor structures $g_{\mu \nu}$ and $g_{\mu \alpha} g_{\nu \beta}+g_{\mu \beta} g_{\nu \alpha}$ for analysis, thus we separate the contributions of the $\frac{3}{2}^{ \pm}$and $\frac{5}{2}^{ \pm}$pentaquark states unambiguously, and tentatively assign $P_{c}(4380)$ and $P_{c}(4450)$ to be the $\frac{3}{2}^{-}$and $\frac{5}{2}^{+}$pentaquark states, respectively.

The current $J_{\mu}(x)$ has non-vanishing couplings with the scattering states $p J / \psi, \Lambda_{c}^{+} \bar{D}^{* 0}, p \chi_{c 1}$ etc. In the following, we illustrate how to take into account the contributions of the intermediate baryon-meson loops to the correlation function $\Pi_{\mu \nu}(p)$,

$$
\begin{aligned}
\Pi_{\mu \nu}(p) & \frac{1}{p-\widehat{M}_{-}-\Sigma_{p J / \psi}^{-}(p)-\Sigma_{\Lambda_{c}^{+} \bar{D}^{* 0}}^{-}(p)-\Sigma_{p \chi_{c 1}}^{-}(p)+\cdots} \lambda_{\frac{3}{2}}^{-2} g_{\mu \nu} \\
& +i \gamma_{5} \frac{1}{p-\widehat{M}_{+}-\Sigma_{p J / \psi}^{+}(p)-\Sigma_{\Lambda_{c}^{+} \bar{D}^{* 0}}^{+}(p)-\Sigma_{p \chi_{c 1}}^{+}(p)+\cdots} \\
& \times i \gamma_{5} \lambda_{\frac{3}{2}}^{+2} g_{\mu \nu}+\cdots,
\end{aligned}
$$

where $\lambda_{\frac{3}{2}}^{ \pm}$and $\widehat{M}_{ \pm}$are bare quantities to absorb the divergences in the self-energies $\Sigma_{p J / \psi}^{ \pm}(p), \Sigma_{\Lambda_{c}^{+} \bar{D}^{* 0}}^{ \pm}(p), \Sigma_{p \chi_{c 1}}^{ \pm}(p)$, etc. The renormalized self-energies contribute a finite imaginary part to modify the dispersion relation,

$$
\begin{aligned}
\Pi_{\mu \nu}(p)= & \frac{p+M_{-}}{p^{2}-M_{-}^{2}+i \sqrt{p^{2}} \Gamma_{-}\left(p^{2}\right)} \lambda_{\frac{3}{2}}^{-2} g_{\mu \nu} \\
& +\frac{p-M_{+}}{p^{2}-M_{+}^{2}+i \sqrt{p^{2}} \Gamma_{+}\left(p^{2}\right)} \lambda_{\frac{3}{2}}^{+2} g_{\mu \nu} \cdots .
\end{aligned}
$$

If we assign the $P_{c}(4380)$ to be the $J^{P}=\frac{3}{2}^{-}$pentaquark state, the width obeys $\Gamma_{-}\left(p^{2}=M_{-}^{2}\right)=\Gamma_{P_{c}(4380)}=$ $205 \pm 18 \pm 86 \mathrm{MeV}$, which is much smaller than the width of $Z_{c}(4200), \Gamma_{Z_{c}(4200)}=370_{-70-132}^{+70+70} \mathrm{MeV}$. In Ref. [39], we observe that the finite width (even as large as $400 \mathrm{MeV}$ ) effect can be absorbed into the pole residue $\lambda_{Z_{c}(4200)}$ safely, the intermediate meson loops cannot affect the mass $M_{Z_{c}(4200)}$ significantly, so the zero width approximation in the hadronic spectral density works. The contributions of the intermediate baryon-meson loops to the correlation function $\Pi_{\mu \nu \alpha \beta}(p)$ can be studied analogously, furthermore, the width $\Gamma_{P_{c}(4450)}$ is much smaller than the width $\Gamma_{P_{c}(4380)}$. In this article, we take the zero width approximation, which will not impair the predictive ability significantly.

Now we obtain the spectral densities at the phenomenological side through the dispersion relation,

$$
\begin{aligned}
\frac{\operatorname{Im} \Pi_{\frac{3}{2}}(s)}{\pi}= & p\left[\lambda_{\frac{3}{2}}^{-2} \delta\left(s-M_{-}^{2}\right)+\lambda_{\frac{3}{2}}^{+2} \delta\left(s-M_{+}^{2}\right)\right] \\
& +\left[M_{-} \lambda_{\frac{3}{2}}^{-2} \delta\left(s-M_{-}^{2}\right)-M_{+} \lambda_{\frac{3}{2}}^{+2} \delta\left(s-M_{+}^{2}\right)\right], \\
= & p \rho_{\frac{3}{2}}^{1}, H(s)+\rho_{\frac{3}{2}, H}^{0}(s),
\end{aligned}
$$

$\frac{\operatorname{Im} \Pi_{\frac{5}{2}}(s)}{\pi}=\not p\left[\lambda_{\frac{5}{2}}^{+2} \delta\left(s-M_{+}^{2}\right)+\lambda_{\frac{5}{2}}^{-2} \delta\left(s-M_{-}^{2}\right)\right]$ 


$$
\begin{aligned}
& +\left[M_{+} \lambda_{\frac{5}{2}}^{+2} \delta\left(s-M_{+}^{2}\right)-M_{-} \lambda_{\frac{5}{2}}^{-2} \delta\left(s-M_{-}^{2}\right)\right], \\
= & \not p \rho_{\frac{5}{2}, H}^{1}(s)+\rho_{\frac{5}{2}, H}^{0}(s),
\end{aligned}
$$

where the subscript $H$ denotes the hadron side, then we introduce the weight function $\exp \left(-\frac{s}{T^{2}}\right)$ to obtain the QCD sum rules at the phenomenological side (or the hadron side),

$$
\begin{gathered}
\int_{4 m_{c}^{2}}^{s_{0}} \mathrm{~d} s\left[\sqrt{s} \rho_{\frac{3}{2}, H}^{1}(s)+\rho_{\frac{3}{2}, H}^{0}(s)\right] \exp \left(-\frac{s}{T^{2}}\right) \\
=2 M_{-} \lambda_{\frac{3}{2}}^{-2} \exp \left(-\frac{M_{-}^{2}}{T^{2}}\right), \\
\int_{4 m_{c}^{2}}^{s_{0}} \mathrm{~d} s\left[\sqrt{s} \rho_{\frac{3}{2}, H}^{1}(s)-\rho_{\frac{3}{2}, H}^{0}(s)\right] \exp \left(-\frac{s}{T^{2}}\right) \\
=2 M_{+} \lambda_{\frac{3}{2}}^{+2} \exp \left(-\frac{M_{+}^{2}}{T^{2}}\right), \\
\int_{4 m_{c}^{2}}^{s_{0}} \mathrm{~d} s\left[\sqrt{s} \rho_{\frac{5}{2}, H}^{1}(s)+\rho_{\frac{5}{2}, H}^{0}(s)\right] \exp \left(-\frac{s}{T^{2}}\right) \\
=2 M_{+} \lambda_{\frac{5}{2}}^{+2} \exp \left(-\frac{M_{+}^{2}}{T^{2}}\right), \\
\int_{4 m_{c}^{2}}^{s_{0}} \mathrm{~d} s\left[\sqrt{s} \rho_{\frac{5}{2}, H}^{1}(s)-\rho_{\frac{5}{2}, H}^{0}(s)\right] \exp \left(-\frac{s}{T^{2}}\right) \\
=2 M_{-} \lambda_{\frac{5}{2}}^{-2} \exp \left(-\frac{M_{-}^{2}}{T^{2}}\right),
\end{gathered}
$$

where the $s_{0}$ are the continuum threshold parameters and the $T^{2}$ are the Borel parameters. We separate the contributions of the negative parity pentaquark states from that of the positive parity pentaquark states unambiguously.

In the following, we briefly outline the operator product expansion for the correlation functions $\Pi_{\mu \nu}(p)$ and $\Pi_{\mu \nu \alpha \beta}(p)$ in perturbative QCD. We contract the $u, d$, and $c$ quark fields in the correlation functions $\Pi_{\mu \nu}(p)$ and $\Pi_{\mu \nu \alpha \beta}(p)$ with the Wick theorem, and obtain the results:

$$
\begin{aligned}
\Pi_{\mu \nu}(p)= & i \varepsilon^{i l a} \varepsilon^{i j k} \varepsilon^{l m n} \varepsilon^{i^{\prime} l^{\prime} a^{\prime}} \varepsilon^{i^{\prime} j^{\prime} k^{\prime}} \varepsilon^{l^{\prime} m^{\prime} n^{\prime}} \int \mathrm{d}^{4} x e^{i p \cdot x} \\
& \times\left\{\operatorname{Tr}\left[\gamma_{5} D_{k k^{\prime}}(x) \gamma_{5} C U_{j j^{\prime}}^{T}(x) C\right]\right. \\
& \times \operatorname{Tr}\left[\gamma_{\mu} C_{n n^{\prime}}(x) \gamma_{\nu} C U_{m m^{\prime}}^{T}(x) C\right] C C_{a^{\prime} a}^{T}(-x) C \\
& -\operatorname{Tr}\left[\gamma_{5} D_{k k^{\prime}}(x) \gamma_{5} C U_{m j^{\prime}}^{T}(x) C \gamma_{\mu}\right. \\
& \left.\left.\times C_{n n^{\prime}}(x) \gamma_{\nu} C U_{j m^{\prime}}^{T}(x) C\right] C C_{a^{\prime} a}^{T}(-x) C\right\}, \quad(35) \\
\Pi_{\mu \nu \alpha \beta}(p)= & \frac{i}{2} \varepsilon^{i l a} \varepsilon^{i j k} \varepsilon^{l m n} \varepsilon^{i^{\prime} l^{\prime} a^{\prime}} \varepsilon^{i^{\prime} j^{\prime} k^{\prime}} \varepsilon^{l^{\prime} m^{\prime} n^{\prime}} \int \mathrm{d}^{4} x e^{i p \cdot x} \\
& \times\left\{\operatorname{Tr}\left[\gamma_{5} D_{k k^{\prime}}(x) \gamma_{5} C U_{j j^{\prime}}^{T}(x) C\right]\right. \\
& \times \operatorname{Tr}\left[\gamma_{\mu} C_{n n^{\prime}}(x) \gamma_{\alpha} C U_{m m^{\prime}}^{T}(x) C\right] \gamma_{\nu} C C_{a^{\prime} a}^{T}(-x) C \gamma_{\beta} \\
& +\operatorname{Tr}\left[\gamma_{5} D_{k k^{\prime}}(x) \gamma_{5} C U_{j j^{\prime}}^{T}(x) C\right]
\end{aligned}
$$

$$
\begin{aligned}
& \times \operatorname{Tr}\left[\gamma_{\nu} C_{n n^{\prime}}(x) \gamma_{\alpha} C U_{m m^{\prime}}^{T}(x) C\right] \gamma_{\mu} C C_{a^{\prime} a}^{T}(-x) C \gamma_{\beta} \\
& +\operatorname{Tr}\left[\gamma_{5} D_{k k^{\prime}}(x) \gamma_{5} C U_{j j^{\prime}}^{T}(x) C\right] \\
& \times \operatorname{Tr}\left[\gamma_{\mu} C_{n n^{\prime}}(x) \gamma_{\beta} C U_{m m^{\prime}}^{T}(x) C\right] \gamma_{\nu} C C_{a^{\prime} a}^{T}(-x) C \gamma_{\alpha} \\
& +\operatorname{Tr}\left[\gamma_{5} D_{k k^{\prime}}(x) \gamma_{5} C U_{j j^{\prime}}^{T}(x) C\right] \\
& \times \operatorname{Tr}\left[\gamma_{\nu} C_{n n^{\prime}}(x) \gamma_{\beta} C U_{m m^{\prime}}^{T}(x) C\right] \gamma_{\mu} C C_{a^{\prime} a}^{T}(-x) C \gamma_{\alpha} \\
& -\operatorname{Tr}\left[\gamma_{5} D_{k k^{\prime}}(x) \gamma_{5} C U_{m j^{\prime}}^{T}(x) C \gamma_{\mu} C_{n n^{\prime}}(x) \gamma_{\alpha} C U_{j m^{\prime}}^{T}(x) C\right] \\
& \times \gamma_{\nu} C C_{a^{\prime} a}^{T}(-x) C \gamma_{\beta} \\
& -\operatorname{Tr}\left[\gamma_{5} D_{k k^{\prime}}(x) \gamma_{5} C U_{m j^{\prime}}^{T}(x) C \gamma_{\nu} C_{n n^{\prime}}(x) \gamma_{\alpha} C U_{j m^{\prime}}^{T}(x) C\right] \\
& \times \gamma_{\mu} C C_{a^{\prime} a}^{T}(-x) C \gamma_{\beta} \\
& -\operatorname{Tr}\left[\gamma_{5} D_{k k^{\prime}}(x) \gamma_{5} C U_{m j^{\prime}}^{T}(x) C \gamma_{\mu} C_{n n^{\prime}}(x) \gamma_{\beta} C U_{j m^{\prime}}^{T}(x) C\right] \\
& \times \gamma_{\nu} C C_{a^{\prime} a}^{T}(-x) C \gamma_{\alpha} \\
& -\operatorname{Tr}\left[\gamma_{5} D_{k k^{\prime}}(x) \gamma_{5} C U_{m j^{\prime}}^{T}(x) C \gamma_{\nu} C_{n n^{\prime}}(x) \gamma_{\beta} C U_{j m^{\prime}}^{T}(x) C\right] \\
& \left.\times \gamma_{\mu} C C_{a^{\prime} a}^{T}(-x) C \gamma_{\alpha}\right\},
\end{aligned}
$$

where $U_{i j}(x), D_{i j}(x)$, and $C_{i j}(x)$ are the full $u, d$, and $c$ quark propagators, respectively $\left(S_{i j}(x)=U_{i j}(x), D_{i j}(x)\right)$,

$$
\begin{aligned}
S_{i j}(x)= & \frac{i \delta_{i j} \not x}{2 \pi^{2} x^{4}}-\frac{\delta_{i j}\langle\bar{q} q\rangle}{12}-\frac{\delta_{i j} x^{2}\left\langle\bar{q} g_{s} \sigma G q\right\rangle}{192} \\
& -\frac{i g_{s} G_{\alpha \beta}^{a} t_{i j}^{a}\left(\not x \sigma^{\alpha \beta}+\sigma^{\alpha \beta} \not x\right)}{32 \pi^{2} x^{2}} \\
& -\frac{1}{8}\left\langle\bar{q}_{j} \sigma^{\mu \nu} q_{i}\right\rangle \sigma_{\mu \nu}+\cdots,
\end{aligned}
$$

$$
\begin{aligned}
C_{i j}(x)= & \frac{i}{(2 \pi)^{4}} \int d^{4} k e^{-i k \cdot x} \\
& \times\left\{\frac{\delta_{i j}}{\not k-m_{c}}-\frac{g_{s} G_{\alpha \beta}^{n} t_{i j}^{n}}{4} \frac{\sigma^{\alpha \beta}\left(\not k+m_{c}\right)+\left(\not k+m_{c}\right) \sigma^{\alpha \beta}}{\left(k^{2}-m_{c}^{2}\right)^{2}}\right. \\
& \left.-\frac{g_{s}^{2}\left(t^{a} t^{b}\right)_{i j} G_{\alpha \beta}^{a} G_{\mu \nu}^{b}\left(f^{\alpha \beta \mu \nu}+f^{\alpha \mu \beta \nu}+f^{\alpha \mu \nu \beta}\right)}{4\left(k^{2}-m_{c}^{2}\right)^{5}}+\cdots\right\}, \\
f^{\alpha \beta \mu \nu}= & \left(\not k+m_{c}\right) \gamma^{\alpha}\left(\not k+m_{c}\right) \gamma^{\beta}\left(\not k+m_{c}\right) \\
& \times \gamma^{\mu}\left(\not k+m_{c}\right) \gamma^{\nu}\left(\not k+m_{c}\right),
\end{aligned}
$$

and $t^{n}=\frac{\lambda^{n}}{2}$ and $\lambda^{n}$ is for the Gell-Mann matrices [56], then we compute the integrals both in the coordinate and momentum spaces to obtain the correlation functions $\Pi_{\mu \nu}(p)$ and $\Pi_{\mu \nu \alpha \beta}(p)$, and therefore the QCD spectral densities $\rho_{\frac{3}{2} / \frac{5}{2}, \mathrm{QCD}}^{1}(s)$ and $\rho_{\frac{3}{2} / \frac{5}{2}, \mathrm{QCD}}^{0}(s)$ through the dispersion relation. In Eq. (37), we retain the term $\left\langle\bar{q}_{j} \sigma_{\mu \nu} q_{i}\right\rangle$, which comes from the Fierz re-arrangement of $\left\langle q_{i} \bar{q}_{j}\right\rangle$ to absorb the gluons emitted from both the heavy quark lines and the light quark lines to form $\left\langle\bar{q}_{j} g_{s} G_{\alpha \beta}^{a} t_{m n}^{a} \sigma_{\mu \nu} q_{i}\right\rangle$ so as to extract the mixed condensate $\left\langle\bar{q} g_{s} \sigma G q\right\rangle$.

Once the analytical QCD spectral densities $\rho_{\frac{3}{2} / \frac{5}{2}, \mathrm{QCD}}^{1}(s)$ and $\rho_{\frac{3}{2} / \frac{5}{2}}^{0}$,QCD $(s)$ are obtained, we can take the quark-hadron duality below the continuum thresholds $s_{0}$ and introduce the 
weight function $\exp \left(-\frac{s}{T^{2}}\right)$ to obtain the following QCD sum rules:

$$
\begin{aligned}
& 2 M_{-} \lambda_{\frac{3}{2}}^{-2} \exp \left(-\frac{M_{-}^{2}}{T^{2}}\right) \\
& =\int_{4 m_{c}^{2}}^{s_{0}} \mathrm{~d} s\left[\sqrt{s} \rho_{\frac{3}{2}, \mathrm{QCD}}^{1}(s)+\rho_{\frac{3}{2}, \mathrm{QCD}}^{0}(s)\right] \exp \left(-\frac{s}{T^{2}}\right) \\
& 2 M_{+} \lambda_{\frac{3}{2}}^{+2} \exp \left(-\frac{M_{+}^{2}}{T^{2}}\right) \\
& =\int_{4 m_{c}^{2}}^{s_{0}} \mathrm{~d} s\left[\sqrt{s} \rho_{\frac{3}{2}, \mathrm{QCD}}^{1}(s)-\rho_{\frac{3}{2}, \mathrm{QCD}}^{0}(s)\right] \exp \left(-\frac{s}{T^{2}}\right) \\
& 2 M_{+} \lambda_{\frac{5}{2}}^{+2} \exp \left(-\frac{M_{+}^{2}}{T^{2}}\right) \\
& =\int_{4 m_{c}^{2}}^{s_{0}} \mathrm{~d} s\left[\sqrt{s} \rho_{\frac{5}{2}, \mathrm{QCD}}^{1}(s)-\rho_{\frac{5}{2}, \mathrm{QCD}}^{0}(s)\right] \exp \left(-\frac{s}{T^{2}}\right), \\
& 2 M_{-} \lambda_{\frac{5}{2}}^{-2} \exp \left(-\frac{M_{-}^{2}}{T^{2}}\right) \\
& =\int_{4 m_{c}^{2}}^{s_{0}} \mathrm{~d} s\left[\sqrt{s} \rho_{\frac{5}{2}, \mathrm{QCD}}^{1}(s)+\rho_{\frac{5}{2}, \mathrm{QCD}}^{0}(s)\right] \exp \left(-\frac{s}{T^{2}}\right)
\end{aligned}
$$

where

$$
\begin{aligned}
\rho_{\frac{3}{2}, \mathrm{QCD}}^{1}(s)= & \rho_{\mathrm{QCD}}^{1}(s), \\
\rho_{\frac{5}{2}, \mathrm{QCD}}^{1}(s)= & 2 \rho_{\mathrm{QCD}}^{1}(s), \\
\rho_{\frac{3}{2}, \mathrm{QCD}}^{0}(s)= & m_{c} \widetilde{\rho}_{\mathrm{QCD}}^{0}(s), \\
\rho_{\frac{5}{2}, \mathrm{QCD}}^{0}(s)= & 2 m_{c} \widetilde{\rho}_{\mathrm{QCD}}^{0}(s), \\
\rho_{\mathrm{QCD}}^{1}(s)= & \rho_{0}^{1}(s)+\rho_{3}^{1}(s)+\rho_{4}^{1}(s)+\rho_{5}^{1}(s)+\rho_{6}^{1}(s) \\
& +\rho_{8}^{1}(s)+\rho_{9}^{1}(s)+\rho_{10}^{1}(s), \\
\widetilde{\rho}_{\mathrm{QCD}}^{0}(s)= & \widetilde{\rho}_{0}^{0}(s)+\widetilde{\rho}_{3}^{0}(s)+\widetilde{\rho}_{4}^{0}(s)+\widetilde{\rho}_{5}^{0}(s)+\widetilde{\rho}_{6}^{0}(s) \\
& +\widetilde{\rho}_{8}^{0}(s)+\widetilde{\rho}_{9}^{0}(s)+\widetilde{\rho}_{10}^{0}(s),
\end{aligned}
$$

the explicit expressions of the QCD spectral densities $\rho_{i}^{1}(s)$ and $\widetilde{\rho}_{i}^{0}(s)$ with $i=0,3,4,5,6,8,9,10$ are shown in the appendix.

From Eqs. (39), (40), (41), (42), (43), and (44), we can see that if we set $\lambda_{\frac{5}{2}}^{+}=\sqrt{2} \lambda_{\frac{3}{2}}^{+}$and $\lambda_{\frac{5}{2}}^{-}=\sqrt{2} \lambda_{\frac{3}{2}}^{-}$, the four QCD sum rules in Eqs. (39), (40), (41), and (42) are reduced to two QCD sum rules, the negative parity pentaquark states have degenerate masses, and the positive parity pentaquark states also have degenerate masses. The LHCb collaboration observe that the best fit leads to the spin-parity assignment $\left(\frac{3}{2}^{-}, \frac{5}{2}^{+}\right)$for the $\left(P_{c}(4380), P_{c}(4450)\right)$, but other assignments, such as $\left(\frac{3}{2}^{+}, \frac{5}{2}^{-}\right)$and $\left(\frac{5}{2}^{+}, \frac{3}{2}^{-}\right)$, are also acceptable [20]. Meanwhile, Eqs. (39), (40), (41), (42), (43), and (44) indicate that the pentaquark states with the spin-parity $\left(\frac{3}{2}^{-}, \frac{5}{2}^{+}\right)$and $\left(\frac{5}{2}^{-}, \frac{3}{2}^{+}\right)$have degenerate masses, which contradicts with the assignments $\left(\frac{3}{2}^{+}, \frac{5}{2}^{-}\right)$and $\left(\frac{5}{2}^{+}, \frac{3}{2}^{-}\right)$.

In this article, we carry out the operator product expansion to the vacuum condensates up to dimension- 10 , and we assume vacuum saturation for the higher dimension vacuum condensates; see Eqs. $(35,36)$, (37), and (38). We take the truncations $n \leq 10$ and $k \leq 1$ in a consistent way, the operators of the orders $\mathcal{O}\left(\alpha_{s}^{k}\right)$ with $k>1$ are discarded. The condensates $\left\langle g_{s}^{3} G G G\right\rangle,\left\langle\frac{\alpha_{s} G G}{\pi}\right\rangle^{2}$, and $\left\langle\frac{\alpha_{s} G G}{\pi}\right\rangle\left\langle\bar{s} g_{s} \sigma G s\right\rangle$ have the dimensions 6,8 , and 9 , respectively, but they are the vacuum expectations of the operators of the order $\mathcal{O}\left(\alpha_{s}^{3 / 2}\right)$, $\mathcal{O}\left(\alpha_{s}^{2}\right), \mathcal{O}\left(\alpha_{s}^{3 / 2}\right)$, respectively. Furthermore, the numerical values of the condensates $\langle\bar{q} q\rangle\left\langle\frac{\alpha_{s}}{\pi} G G\right\rangle$ and $\langle\bar{q} q\rangle^{2}\left\langle\frac{\alpha_{s}}{\pi} G G\right\rangle$ are very small and accompanied by large denominators, and they are neglected safely.

We differentiate Eqs. (39), (40), (41), and (42) with respect to $\frac{1}{T^{2}}$, then eliminate the pole residues $\lambda_{\frac{3}{2}\left(\frac{5}{2}\right)}^{ \pm}$and obtain the QCD sum rules for the masses of the pentaquark states,

$M_{-}^{2}=\frac{\int_{4 m_{c}^{2}}^{s_{0}} \mathrm{~d} s s\left[\sqrt{s} \rho_{\mathrm{QCD}}^{1}(s)+m_{c} \widetilde{\rho}_{\mathrm{QCD}}^{0}(s)\right] \exp \left(-\frac{s}{T^{2}}\right)}{\int_{4 m_{c}^{2}}^{s_{0}} \mathrm{~d} s\left[\sqrt{s} \rho_{\mathrm{QCD}}^{1}(s)+m_{c} \widetilde{\rho}_{\mathrm{QCD}}^{0}(s)\right] \exp \left(-\frac{s}{T^{2}}\right)}$,

$$
M_{+}^{2}=\frac{\int_{4 m_{c}^{2}}^{s_{0}} \mathrm{~d} s s\left[\sqrt{s} \rho_{\mathrm{QCD}}^{1}(s)-m_{c} \widetilde{\rho}_{\mathrm{QCD}}^{0}(s)\right] \exp \left(-\frac{s}{T^{2}}\right)}{\int_{4 m_{c}^{2}}^{s_{0}} \mathrm{~d} s\left[\sqrt{s} \rho_{\mathrm{QCD}}^{1}(s)-m_{c} \widetilde{\rho}_{\mathrm{QCD}}^{0}(s)\right] \exp \left(-\frac{s}{T^{2}}\right)},
$$

where the $M_{-}\left(M_{+}\right)$are the masses of the $J^{P}=\frac{3}{2}^{-}, \frac{5}{2}^{-}$ $\left(\frac{3}{2}^{+}, \frac{5}{2}^{+}\right)$pentaquark states. Once the masses $M_{ \pm}$are obtained, we can take them as input parameters and obtain the pole residues from the QCD sum rules in Eqs. (39), (40), (41), and (42), and the relations $\lambda_{\frac{5}{2}}^{+}=\sqrt{2} \lambda_{\frac{3}{2}}^{+}$and $\lambda_{\frac{5}{2}}^{-}=\sqrt{2} \lambda_{\frac{3}{2}}^{-}$ hold.

\section{Numerical results and discussions}

We take the vacuum condensates to be the standard values $\langle\bar{q} q\rangle=-(0.24 \pm 0.01 \mathrm{GeV})^{3},\left\langle\bar{q} g_{s} \sigma G q\right\rangle=m_{0}^{2}\langle\bar{q} q\rangle$, $m_{0}^{2}=(0.8 \pm 0.1) \mathrm{GeV}^{2},\left\langle\frac{\alpha_{s} G G}{\pi}\right\rangle=(0.33 \mathrm{GeV})^{4}$ at the energy scale $\mu=1 \mathrm{GeV}[55,56]$. The quark condensates and mixed quark condensates evolve with the renormalization group equation, $\langle\bar{q} q\rangle(\mu)=\langle\bar{q} q\rangle(Q)\left[\frac{\alpha_{s}(Q)}{\alpha_{s}(\mu)}\right]^{\frac{4}{9}}$ and $\left\langle\bar{q} g_{s} \sigma G q\right\rangle(\mu)=\left\langle\bar{q} g_{s} \sigma G q\right\rangle(Q)\left[\frac{\alpha_{s}(Q)}{\alpha_{s}(\mu)}\right]^{\frac{2}{27}}$. In the article, we take the $\overline{M S}$ mass $m_{c}\left(m_{c}\right)=(1.275 \pm 0.025) \mathrm{GeV}$ from the 
Particle Data Group [58], and take into account the energyscale dependence of the $\overline{M S}$ mass from the renormalization group equation

$$
\begin{aligned}
& m_{c}(\mu)=m_{c}\left(m_{c}\right)\left[\frac{\alpha_{s}(\mu)}{\alpha_{s}\left(m_{c}\right)}\right]^{\frac{12}{25}}, \\
& \alpha_{s}(\mu)=\frac{1}{b_{0} t}\left[1-\frac{b_{1}}{b_{0}^{2}} \frac{\log t}{t}+\frac{b_{1}^{2}\left(\log ^{2} t-\log t-1\right)+b_{0} b_{2}}{b_{0}^{4} t^{2}}\right],
\end{aligned}
$$

where $t=\log \frac{\mu^{2}}{\Lambda^{2}}, b_{0}=\frac{33-2 n_{f}}{12 \pi}, b_{1}=\frac{153-19 n_{f}}{24 \pi^{2}}, b_{2}=$ $\frac{2857-\frac{5033}{9} n_{f}+\frac{325}{27} n_{f}^{2}}{128 \pi^{3}}, \Lambda=213 \mathrm{MeV}, 296 \mathrm{MeV}$ and $339 \mathrm{MeV}$ for the flavors $n_{f}=5,4$, and 3, respectively [58].

In Refs. [16-19,37-39,46-48], we studied the acceptable energy scales of the QCD spectral densities for the hidden charm (bottom) tetraquark states and molecular (and molecule-like) states in the QCD sum rules in detail for the first time, and we suggested the formula $\mu=$ $\sqrt{M_{X / Y / Z}^{2}-\left(2 \mathbb{M}_{Q}\right)^{2}}$ to determine the energy scales, where $X, Y$, and $Z$ denote the four-quark systems, and $\mathbb{M}_{Q}$ denotes the effective heavy quark masses. The effective mass $\mathbb{M}_{c}=$ $1.8 \mathrm{GeV}$ is the optimal value for the diquark-antidiquark type tetraquark states [16-19,46-48].

In this article, we use the diquark-diquark-antiquark model to construct the currents to interpolate the hidden charm pentaquark states, but there also exists a $\bar{c} c$ quark pair. The hidden charm (or bottom) five-quark systems $q q_{1} q_{2} Q \bar{Q}$ could be described by a double-well potential, just like the four-quark systems $q q^{\prime} Q \bar{Q}$; see Eqs. (3), (4), (5, 6), (7), and (8) and the related discussions in the introduction. The heavy five-quark states are also characterized by the effective heavy quark masses $\mathbb{M}_{Q}$ and the virtuality $V=\sqrt{M_{P_{c}}^{2}-\left(2 \mathbb{M}_{Q}\right)^{2}}$. The QCD sum rules have three typical energy scales $\mu^{2}, T^{2}$, $V^{2}$, we can also take the energy scale, $\mu^{2}=V^{2}=\mathcal{O}\left(T^{2}\right)$ $[16-19,47,48]$. In this article, we can take the analogous formula,

$\mu=\sqrt{M_{P_{c}}^{2}-\left(2 \mathbb{M}_{c}\right)^{2}}$,

with the value $\mathbb{M}_{c}=1.8 \mathrm{GeV}$ to determine the energy scales of the QCD spectral densities [16-19,47,48], and obtain the values $\mu=2.5 \mathrm{GeV}$ and $\mu=2.6 \mathrm{GeV}$ for the hidden charm pentaquark states $P_{c}(4380)$ and $P_{c}(4450)$, respectively. The energy-scale formula can be rewritten as

$M_{P_{c}}^{2}=\left(2 \mathbb{M}_{c}\right)^{2}+\mu^{2}$.

In this article, we choose the Borel parameters $T^{2}$ and the continuum threshold parameters $s_{0}$ to satisfy the following criteria:

1. pole dominance at the phenomenological side;

2. convergence of the operator product expansion;
3. appearance of the Borel platforms;

4. satisfying the energy-scale formula.

In the QCD sum rules for the multiquark states, it is difficult to satisfy the criteria 1 and 2 . In previous work [16$19,46]$, we observed that the pole contributions can be taken as large as (50-70)\% in the QCD sum rules for the diquark-antidiquark type tetraquark states $q q^{\prime} Q \bar{Q}(X, Y, Z)$, if the QCD spectral densities obey the energy-scale formula $\mu=\sqrt{M_{X / Y / Z}^{2}-\left(2 \mathbb{M}_{Q}\right)^{2}}$. The operator product expansion converges more slowly in the QCD sum rules for the pentaquark states $q q_{1} q_{2} Q \bar{Q}$ compared to that for the tetraquark states $q \bar{q}^{\prime} Q \bar{Q}$, so, in this article, we choose smaller pole contributions, about $(50 \pm 10) \%$. For the tetraquark states $q \bar{q}^{\prime} Q \bar{Q}[16-19,46]$, the Borel platforms appear as the minimum values, and the platforms are very flat, but the Borel windows are small, $T_{\max }^{2}-T_{\min }^{2}=0.4 \mathrm{GeV}^{2}$, where $\max$ and min denote the maximum and minimum values, respectively. For the three-quark baryons $q q^{\prime} Q, q Q Q^{\prime}, Q Q^{\prime} Q^{\prime \prime}$ [54,59-63], the Borel platforms do not appear as the minimum values, the predicted masses increase slowly with the increase of the Borel parameter, and we determine the Borel windows by the criteria 1 and 2; the platforms are not very flat. In this article, we also choose small Borel windows $T_{\max }^{2}-T_{\min }^{2}=0.4 \mathrm{GeV}^{2}$,just like in the case of the tetraquark states, and we obtain the platforms by requiring the uncertainties $\frac{\delta M_{P_{c}}}{M_{P_{c}}}$ induced by the Borel parameters to be about $1 \%$.

Now we search for the optimal Borel parameters $T^{2}$ and continuum threshold parameters $s_{0}$ according to the four criteria. The resulting Borel parameters, continuum threshold parameters, energy scales, and pole contributions are shown explicitly in Table 1. Furthermore, the contributions of the vacuum condensates of dimension 10 are less than $5 \%$, the operator product expansion is convergent. So the four criteria of the QCD sum rules are satisfied; we expect to obtain reasonable predictions. From Table 1, we can see

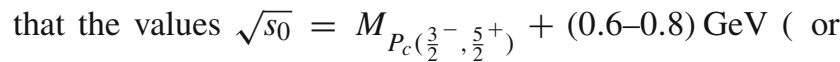
$\left.s_{0}^{P_{c}\left(\frac{3}{2}^{-}\right)}=(26 \pm 1) \mathrm{GeV}^{2}, s_{0}^{P_{c}\left(\frac{5}{2}^{+}\right)}=(27 \pm 1) \mathrm{GeV}^{2}\right) \mathrm{can}$ lead to satisfactory results.

In Fig. 1, we plot the predicted masses with variation of the threshold parameters $s_{0}$, where we assign $P_{c}(4380)$ and $P_{c}(4450)$ to be the $\frac{3}{2}^{-}$and $\frac{5}{2}^{+}$pentaquark states, respectively. From the figure, we can see that the predicted masses increase slowly with (or are not sensitive to) the threshold parameters $s_{0}$ for central values of the other parameters.

In Refs. [54,59-63], we study the $J^{P}=\frac{1}{2}^{ \pm}$and $\frac{3}{2}^{ \pm}$heavy, doubly heavy and triply heavy baryon states systematically with the QCD sum rules by subtracting the contributions from the corresponding $J^{P}=\frac{1}{2}^{\mp}$ and $\frac{3}{2}^{\mp}$ heavy, doubly heavy and triply heavy baryon states. The continuum threshold param- 
Table 1 The Borel parameters, continuum threshold parameters, energy scales, pole contributions, masses, and pole residues of the pentaquark states

\begin{tabular}{lllllll}
\hline & $T^{2}\left(\mathrm{GeV}^{2}\right)$ & $\sqrt{s_{0}}(\mathrm{GeV})$ & $\mu(\mathrm{GeV})$ & Pole $(\%)$ & $M_{P_{c}}(\mathrm{GeV})$ & $\lambda_{P_{c}}\left(\mathrm{GeV}^{6}\right)$ \\
\hline$P_{c}\left(\frac{3}{2}^{-}\right)$ & $3.3-3.7$ & $5.10 \pm 0.10$ & 2.5 & $(40-61)$ & $4.38 \pm 0.13$ & $(1.55 \pm 0.28) \times 10^{-3}$ \\
$P_{c}\left(\frac{5}{2}^{+}\right)$ & $3.1-3.5$ & $5.15 \pm 0.10$ & 2.6 & $(40-63)$ & $4.44 \pm 0.14$ & $(0.84 \pm 0.17) \times 10^{-3}$ \\
\hline
\end{tabular}

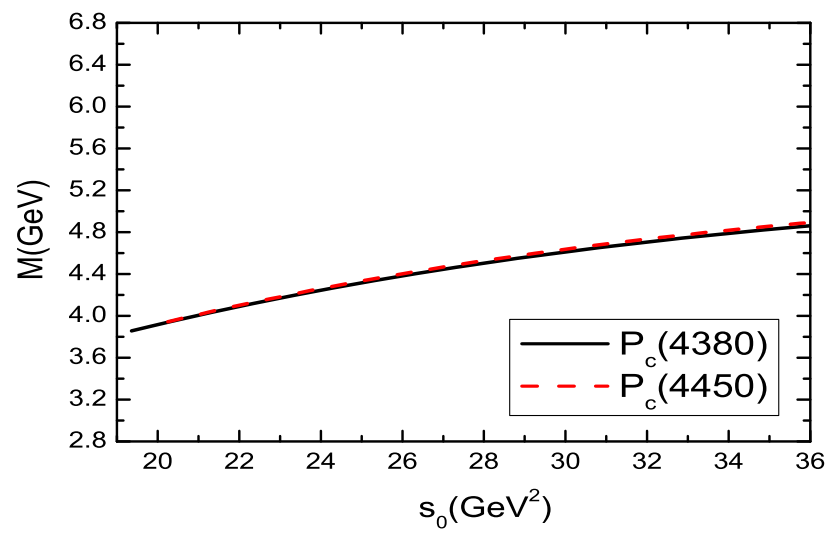

Fig. 1 The masses of the pentaquark states with variations of the threshold parameters $s_{0}$

eters $\sqrt{s_{0}}=M_{\mathrm{gr}}+(0.6-0.8) \mathrm{GeV}$ work well, where the subscript gr denotes the ground states. In the present case, the hidden charm pentaquark states carry a baryon number of one, i.e. they are doubly heavy baryons. So the threshold parameters $\left.\sqrt{s_{0}}=M_{P_{c}\left(\frac{3}{2}{ }^{-}, 5^{+}\right.}{ }^{+}\right)+(0.6-0.8) \mathrm{GeV}$ make sense. One may worry that there exist some contaminations from the higher resonances. The upper bounds of the factors $\exp \left(-\frac{s_{0}}{T^{2}}\right)$ are about 0.0007 and 0.0004 in the QCD sum rules for $P_{c}(4380)$ and $P_{c}(4450)$, respectively, if we take the largest values of the continuum threshold parameters; so the contaminations are greatly suppressed and can be neglected safely.

We take into account all uncertainties of the input parameters, and obtain the values of the masses and pole residues of the $\frac{3}{2}^{-}$and $\frac{5}{2}^{+}$hidden charm pentaquark states, which are shown in Figs. 2, 3 and Table 1. The QCD sum rules in Eqs. (39), (40), (41), (42), (46), and (47) indicate that the pentaquark states with the spin-parity $\left(\frac{3}{2}^{-}, \frac{5}{2}^{+}\right)$and $\left(\frac{5}{2}^{-}, \frac{3}{2}^{+}\right)$ have degenerate masses, and $\lambda_{\frac{5}{2}}^{+}=\sqrt{2} \lambda_{\frac{3}{2}}^{+}$and $\lambda_{\frac{5}{2}}^{-}=\sqrt{2} \lambda_{\frac{3}{2}}^{-}$. Naively, we expect that additionally one unit spin or P-wave can lead to larger masses, so $M_{\frac{5}{2}}{ }^{+}>M_{\frac{3}{2}}{ }^{-}$, while the relation $M_{\frac{3}{2}}+>M_{\frac{5}{2}}$ - needs detailed and refined analysis to obtain the answer "yes" or "no". It is sensible to assign $P_{c}(4380)$ and $P_{c}(4450)$ to be the $\frac{3}{2}^{-}$and $\frac{5}{2}^{+}$pentaquark states, respectively. However, the assignment $\left(\frac{5}{2}^{-}, \frac{3}{2}^{+}\right)$of $\left(P_{c}(4380), P_{c}(4450)\right)$ is not excluded.
From Table 1, we can see that the present predictions $M_{P_{c}(4380)}=4.38 \pm 0.13 \mathrm{GeV}$ and $M_{P_{c}(4450)}=4.44 \pm$ $0.14 \mathrm{GeV}$ are in good agreement with the experimental data of the LHCb collaboration, $M_{P_{c}(4380)}=4380 \pm 8 \pm 29 \mathrm{MeV}$ and $M_{P_{c}(4450)}=4449.8 \pm 1.7 \pm 2.5 \mathrm{MeV}$ [20]. The present predictions support assigning $P_{c}(4380)$ and $P_{c}(4450)$ to be the $\frac{3}{2}^{-}$and $\frac{5}{2}^{+}$hidden charm pentaquark states, respectively, which are consistent with the assignments that $P_{c}(4380)$ and $P_{c}(4450)$ are diquark-diquark-antiquark type pentaquark states [27-29] or the diquark-triquark type pentaquark states [30].

In this article, we take the energy-scale formula $\mu=$ $\sqrt{M_{P_{c}}^{2}-\left(2 \mathbb{M}_{c}\right)^{2}}$ to determine the energy scales of the QCD spectral densities. The pole contributions are about (40$60) \%$, and the contributions of the vacuum condensates of dimension 10 are less than $5 \%$, the two criteria (pole dominance at the phenomenological side and convergence of the operator product expansion) of the conventional QCD sum rules can be satisfied, so we expect to make reasonable predictions. In subsequent work, we shall extend the present work to study the $\frac{1}{2}^{ \pm}$and $\frac{3}{2}^{ \pm}$hidden charm pentaquark states in a systematic way [64-66], where the energy-scale formula $\mu=\sqrt{M_{P_{c}}^{2}-\left(2 \mathbb{M}_{c}\right)^{2}}$ serves as an additional constraint on the predicted masses. The typical energy scales, which characterize the five-quark systems $q_{1} q_{2} q_{3} c \bar{c}$ and serve as the optimal energy scales of the QCD spectral densities, are not independent of the masses of the five-quark systems $q_{1} q_{2} q_{3} c \bar{c}$. All the predictions can be confronted to the experimental data in the future.

The diquark-diquark-antiquark type current with special quantum numbers couples potentially to special pentaquark states according to the tensor analysis in Eqs. (21), (22), (25), and (26). The current can be re-arranged both in the color and Dirac-spinor spaces, and they can be changed to a current as a special superposition of the color singlet baryonmeson type currents. The baryon-meson type currents couple potentially to the baryon-meson pairs. The diquarkdiquark-antiquark type pentaquark state can be taken as a special superposition of a series of baryon-meson pairs, and this embodies the net effects. The decays to its components (baryon-meson pairs) are Okubo-Zweig-Iizuka superallowed, but the re-arrangements in the color-space are nontrivial [67]. 

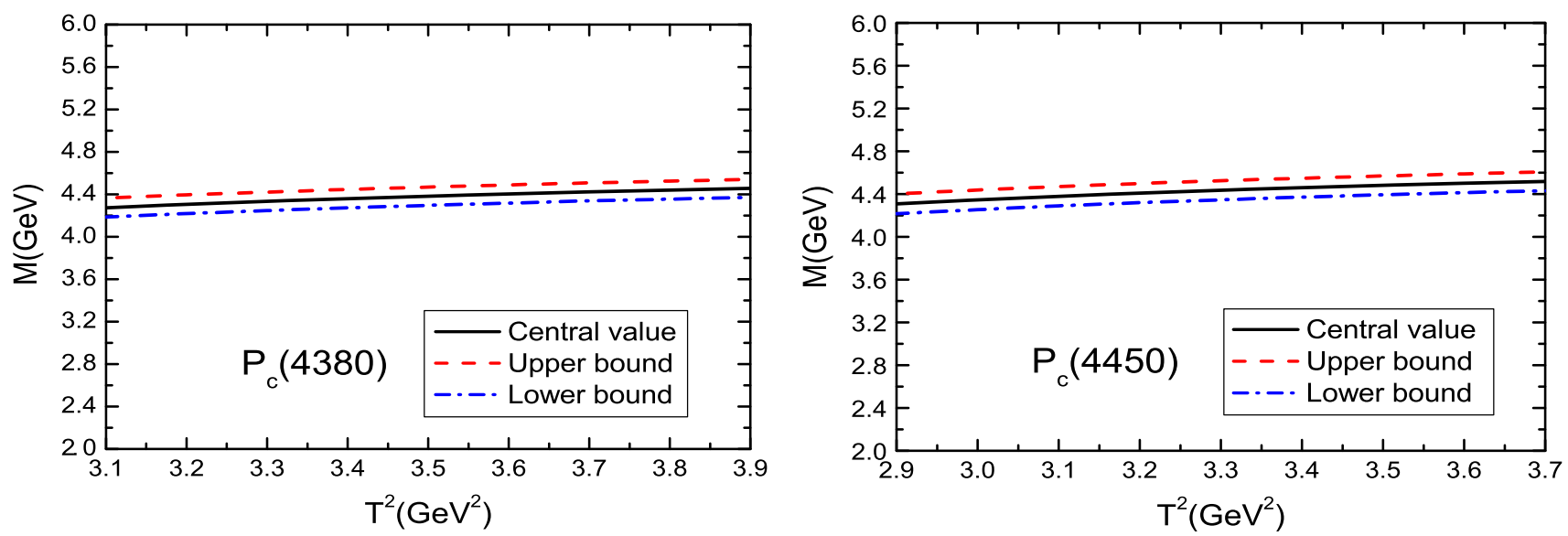

Fig. 2 The masses of the pentaquark states with variations of the Borel parameters $T^{2}$
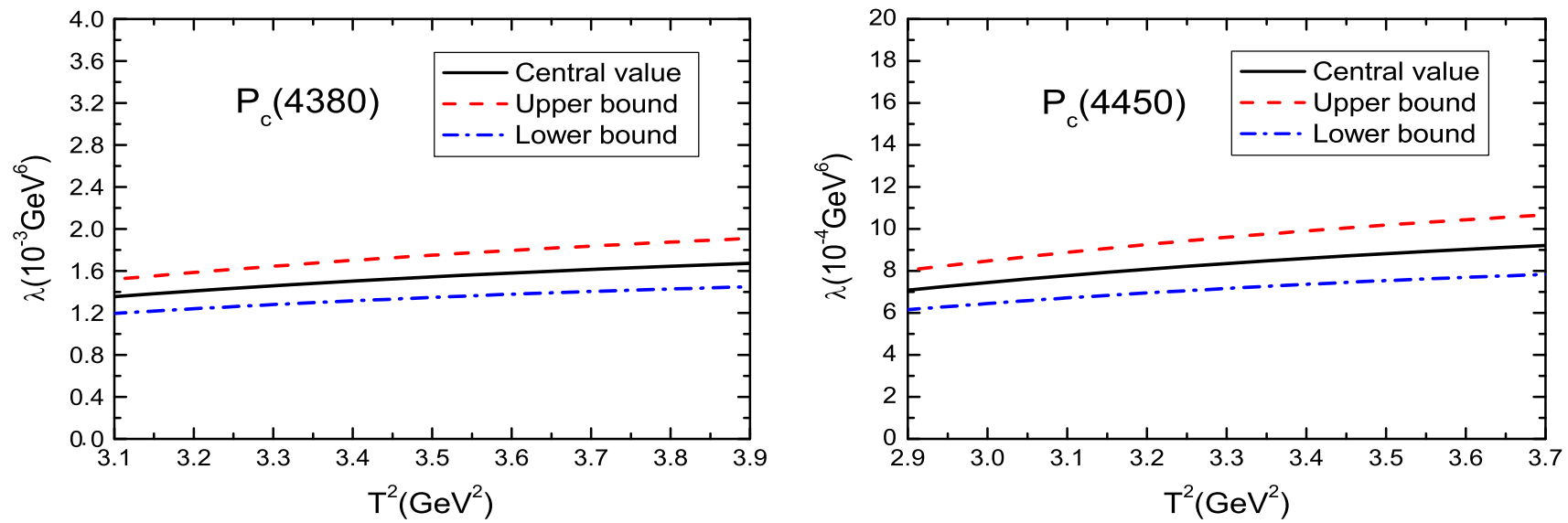

Fig. 3 The pole residues of the pentaquark states with variations of the Borel parameters $T^{2}$

In the following, we perform a Fierz re-arrangement to the currents $J_{\mu}$ and $J_{\mu \nu}$ both in the color and Dirac-spinor spaces to obtain the results,

$$
\begin{aligned}
J_{\mu}= & \frac{1}{4} \mathcal{S} c \bar{c} \gamma_{\mu} u+\frac{1}{4} \mathcal{S} u \bar{c} \gamma_{\mu} c-\frac{1}{4} \mathcal{S} \gamma_{5} c \bar{c} \gamma_{\mu} \gamma_{5} u \\
& -\frac{1}{4} \mathcal{S} \gamma_{5} u \bar{c} \gamma_{\mu} \gamma_{5} c-\frac{i}{4} \mathcal{S} \gamma_{\mu} \gamma_{5} c \bar{c} i \gamma_{5} u-\frac{i}{4} \mathcal{S} \gamma_{\mu} \gamma_{5} u \bar{c} i \gamma_{5} c \\
& -\frac{1}{4} \mathcal{S} \gamma_{\mu} c \bar{c} u-\frac{1}{4} \mathcal{S} \gamma_{\mu} u \bar{c} c-\frac{i}{4} \mathcal{S} \sigma_{\lambda \mu} c \bar{c} \gamma^{\lambda} u \\
& -\frac{i}{4} \mathcal{S} \sigma_{\lambda \mu} u \bar{c} \gamma^{\lambda} c+\frac{i}{4} \mathcal{S} \sigma_{\lambda \mu} \gamma_{5} c \bar{c} \gamma^{\lambda} \gamma_{5} u \\
& +\frac{i}{4} \mathcal{S} \sigma_{\lambda \mu} \gamma_{5} u \bar{c} \gamma^{\lambda} \gamma_{5} c+\frac{1}{8} \mathcal{S} \sigma_{\lambda \tau} \gamma_{\mu} c \bar{c} \sigma^{\lambda \tau} u \\
& +\frac{1}{8} \mathcal{S} \sigma_{\lambda \tau} \gamma_{\mu} u \bar{c} \sigma^{\lambda \tau} c \\
\widehat{J}_{\mu \nu}= & \frac{1}{2 \sqrt{2}} \mathcal{S}\left(g_{\nu \lambda} \gamma_{\mu}+g_{\mu \lambda} \gamma_{\nu}\right) c \bar{c} \gamma^{\lambda} u \\
& +\frac{1}{2 \sqrt{2}} \mathcal{S}\left(g_{\nu \lambda} \gamma_{\mu}+g_{\mu \lambda} \gamma_{\nu}\right) u \bar{c} \gamma^{\lambda} c
\end{aligned}
$$

$$
\begin{aligned}
& -\frac{1}{2 \sqrt{2}} \mathcal{S}\left(g_{\nu \lambda} \gamma_{\mu}+g_{\mu \lambda} \gamma_{\nu}\right) \gamma_{5} c \bar{c} \gamma^{\lambda} \gamma_{5} u \\
& -\frac{1}{2 \sqrt{2}} \mathcal{S}\left(g_{\nu \lambda} \gamma_{\mu}+g_{\mu \lambda} \gamma_{\nu}\right) \gamma_{5} u \bar{c} \gamma^{\lambda} \gamma_{5} c \\
& +\frac{1}{8 \sqrt{2}} \mathcal{S}\left(\gamma_{\mu} \sigma_{\lambda \tau} \gamma_{\nu}+\gamma_{\nu} \sigma_{\lambda \tau} \gamma_{\mu}\right) c \bar{c} \sigma^{\lambda \tau} u \\
& +\frac{1}{8 \sqrt{2}} \mathcal{S}\left(\gamma_{\mu} \sigma_{\lambda \tau} \gamma_{\nu}+\gamma_{\nu} \sigma_{\lambda \tau} \gamma_{\mu}\right) u \bar{c} \sigma^{\lambda \tau} c
\end{aligned}
$$

where we take the replacement $J_{\mu \nu} \rightarrow \widehat{J}_{\mu \nu}$,

$$
\begin{aligned}
J_{\mu \nu} \rightarrow & \widehat{J}_{\mu \nu}, \\
= & \frac{1}{\sqrt{2}} \varepsilon^{i l a} \varepsilon^{i j k} \varepsilon^{l m n} u_{j}^{T} C \gamma_{5} d_{k} \\
& \times\left[u_{m}^{T} C \gamma_{\mu} c_{n} \gamma_{\nu} C \bar{c}_{a}^{T}+u_{m}^{T} C \gamma_{\nu} c_{n} \gamma_{\mu} C \bar{c}_{a}^{T}\right. \\
& \left.-\frac{1}{2} g_{\mu \nu} u_{m}^{T} C \gamma_{\lambda} c_{n} \gamma^{\lambda} C \bar{c}_{a}^{T}\right],
\end{aligned}
$$

to subtract the contribution of the spin- $\frac{1}{2}$ pentaquark state, and we use the notations $\mathcal{S} \Gamma c=\varepsilon^{i j k} u_{i}^{T} C \gamma_{5} d_{j} \Gamma c_{k}$ and 
$\mathcal{S} \Gamma u=\varepsilon^{i j k} u_{i}^{T} C \gamma_{5} d_{j} \Gamma u_{k}$ for simplicity; here $\Gamma$ denotes the Dirac matrices.

The components $\mathcal{S}(x) \Gamma c(x) \bar{c}(x) \Gamma^{\prime} u(x)$ and $\mathcal{S}(x) \Gamma u(x)$ $\bar{c}(x) \Gamma^{\prime} c(x)$ couple potentially to the baryon-meson pairs. The relevant thresholds are $M_{J / \psi p}=4.035 \mathrm{GeV}, M_{\eta_{c} p}=$ $3.922 \mathrm{GeV}, M_{\eta_{c} N(1440)}=4.414 \mathrm{GeV}, M_{\chi_{c 0} p}=4.353 \mathrm{GeV}$, $M_{\Lambda_{c}^{+} \bar{D}^{0}}=4.151 \mathrm{GeV}, M_{\Lambda_{c}^{+} \bar{D}^{* 0}}=4.293 \mathrm{GeV}, M_{h_{c} p}=$ $4.463 \mathrm{GeV}, M_{\chi_{c 1} p}=4.449 \mathrm{GeV}$, and $M_{\Lambda_{c}^{+}(2595) \bar{D}^{0}}=$ $4.457 \mathrm{GeV}$ [58]. After taking into account the current-hadron duality, we obtain the Okubo-Zweig-Iizuka super-allowed decays,

$$
\begin{aligned}
& P_{c}(4380) \rightarrow p J / \psi, \Lambda_{c}^{+} \bar{D}^{* 0}, p \eta_{c}, \Lambda_{c}^{+} \bar{D}^{0}, p \chi_{c 0}, \\
& P_{c}(4450) \rightarrow p J / \psi, \Lambda_{c}^{+} \bar{D}^{* 0}, p \eta_{c}, \Lambda_{c}^{+} \bar{D}^{0}, N(1440) \eta_{c} .
\end{aligned}
$$

We can search for $P_{c}(4380)$ and $P_{c}$ (4450) in the $\Lambda_{c}^{+} \bar{D}^{* 0}$, $p \eta_{c}, \Lambda_{c}^{+} \bar{D}^{0}, p \chi_{c 0}$, and $N(1440) \eta_{c}$ mass distributions in the future, which may shed light on the nature of those pentaquark states.

\section{Conclusion}

In this article, we construct the diquark-diquark-antiquark type interpolating currents, and we study the masses and pole residues of the $\frac{3}{2}^{-}$and $\frac{5}{2}^{+}$hidden charm pentaquark states in detail with the QCD sum rules by calculating the contributions of the vacuum condensates up to dimension-10 in the operator product expansion. In calculations, we use the formula $\mu=\sqrt{M_{P_{c}}^{2}-\left(2 \mathbb{M}_{c}\right)^{2}}$ to determine the energy scales of the QCD spectral densities. The present predictions favor assigning $P_{c}(4380)$ and $P_{c}(4450)$ to be the $\frac{3}{2}^{-}$and $\frac{5}{2}^{+}$pentaquark states, respectively. The pole residues can be taken as basic input parameters to study relevant processes of the pentaquark states with the three-point QCD sum rules.

Acknowledgments This work is supported by National Natural Science Foundation, Grant Numbers 11375063, and Natural Science Foundation of Hebei province, Grant Number A2014502017.

Open Access This article is distributed under the terms of the Creative Commons Attribution 4.0 International License (http://creativecomm ons.org/licenses/by/4.0/), which permits unrestricted use, distribution, and reproduction in any medium, provided you give appropriate credit to the original author(s) and the source, provide a link to the Creative Commons license, and indicate if changes were made. Funded by SCOAP 3 .

\section{Appendix}

For the QCD spectral densities $\rho_{i}^{1}(s)$ and $\widetilde{\rho}_{i}^{0}(s)$ with $i=$ $0,3,4,5,6,8,9,10$ of the pentaquark states, we have

$$
\begin{aligned}
\rho_{0}^{1}(s)= & \frac{1}{491520 \pi^{8}} \int \mathrm{d} y \mathrm{~d} z y z(1-y-z)^{4}\left(s-\bar{m}_{c}^{2}\right)^{4} \\
& \times\left(7 s-2 \bar{m}_{c}^{2}\right), \\
\widetilde{\rho}_{0}^{0}(s)= & \frac{1}{983040 \pi^{8}} \int \mathrm{d} y \mathrm{~d} z(y+z)(1-y-z)^{4}\left(s-\bar{m}_{c}^{2}\right)^{4} \\
& \times\left(6 s-\bar{m}_{c}^{2}\right), \\
\rho_{3}^{1}(s)= & -\frac{m_{c}\langle\bar{q} q\rangle}{3072 \pi^{6}} \int \mathrm{d} y \mathrm{~d} z(y+z)(1-y-z)^{2}\left(s-\bar{m}_{c}^{2}\right)^{3}, \\
\widetilde{\rho}_{3}^{0}(s)= & -\frac{m_{c}\langle\bar{q} q\rangle}{1536 \pi^{6}} \int \mathrm{d} y \mathrm{~d} z(1-y-z)^{2}\left(s-\bar{m}_{c}^{2}\right)^{3},
\end{aligned}
$$

$\rho_{4}^{1}(s)=-\frac{m_{c}^{2}}{73728 \pi^{6}}\left\langle\frac{\alpha_{s} G G}{\pi}\right\rangle \int \mathrm{d} y \mathrm{~d} z\left(\frac{z}{y^{2}}+\frac{y}{z^{2}}\right)$ $\times(1-y-z)^{4}\left(s-\bar{m}_{c}^{2}\right)\left(2 s-\bar{m}_{c}^{2}\right)$

$-\frac{19}{7077888 \pi^{6}}\left\langle\frac{\alpha_{s} G G}{\pi}\right\rangle \int \mathrm{d} y \mathrm{~d} z(y+z)(1-y-z)^{3}$

$\times\left(s-\bar{m}_{c}^{2}\right)^{2}\left(7 s-4 \bar{m}_{c}^{2}\right)$

$+\frac{13}{393216 \pi^{6}}\left\langle\frac{\alpha_{s} G G}{\pi}\right\rangle \int \mathrm{d} y \mathrm{~d} z y z(1-y-z)^{2}$

$\times\left(s-\bar{m}_{c}^{2}\right)^{2}\left(5 s-2 \bar{m}_{c}^{2}\right)$,

$\widetilde{\rho}_{4}^{0}(s)=-\frac{m_{c}^{2}}{294912 \pi^{6}}\left\langle\frac{\alpha_{s} G G}{\pi}\right\rangle \int \mathrm{d} y \mathrm{~d} z\left(\frac{1}{y^{2}}+\frac{1}{z^{2}}+\frac{y}{z^{3}}+\frac{z}{y^{3}}\right)$

$\times(1-y-z)^{4}\left(s-\bar{m}_{c}^{2}\right)\left(3 s-\bar{m}_{c}^{2}\right)$

$+\frac{1}{294912 \pi^{6}}\left\langle\frac{\alpha_{s} G G}{\pi}\right\rangle \int \mathrm{d} y \mathrm{~d} z\left(\frac{y}{z^{2}}+\frac{z}{y^{2}}\right)$

$\times(1-y-z)^{4}\left(s-\bar{m}_{c}^{2}\right)^{2}\left(4 s-\bar{m}_{c}^{2}\right)$

$-\frac{19}{1179648 \pi^{6}}\left\langle\frac{\alpha_{s} G G}{\pi}\right\rangle \int \mathrm{d} y \mathrm{~d} z(1-y-z)^{3}$

$\times\left(s-\bar{m}_{c}^{2}\right)^{2}\left(2 s-\bar{m}_{c}^{2}\right)$

$+\frac{13}{786432 \pi^{6}}\left\langle\frac{\alpha_{s} G G}{\pi}\right\rangle \int \mathrm{d} y \mathrm{~d} z(y+z)(1-y-z)^{2}$

$\times\left(s-\bar{m}_{c}^{2}\right)^{2}\left(4 s-\bar{m}_{c}^{2}\right)$,

$$
\begin{aligned}
\rho_{5}^{1}(s)= & \frac{m_{c}\left\langle\bar{q} g_{s} \sigma G q\right\rangle}{2048 \pi^{6}} \int \mathrm{d} y \mathrm{~d} z(y+z)(1-y-z)\left(s-\bar{m}_{c}^{2}\right)^{2} \\
& +\frac{m_{c}\left\langle\bar{q} g_{s} \sigma G q\right\rangle}{65536 \pi^{6}} \int \mathrm{d} y \mathrm{~d} z \\
& \times\left(\frac{y}{z}+\frac{z}{y}\right)(1-y-z)^{2}\left(s-\bar{m}_{c}^{2}\right)^{2} \\
& -\frac{m_{c}\left\langle\bar{q} g_{s} \sigma G q\right\rangle}{98304 \pi^{6}} \int \mathrm{d} y \mathrm{~d} z\left(\frac{y}{z}+\frac{z}{y}\right)
\end{aligned}
$$




$$
\begin{aligned}
& \times(1-y-z)^{3}\left(s-\bar{m}_{c}^{2}\right)^{2} \\
& +\frac{3 m_{c}\left\langle\bar{q} g_{s} \sigma G q\right\rangle}{32768 \pi^{6}} \int \mathrm{d} y \mathrm{~d} z(y+z) \\
& \times(1-y-z)\left(s-\bar{m}_{c}^{2}\right)^{2}, \\
\widetilde{\rho}_{5}^{0}(s)= & \frac{m_{c}\left\langle\bar{q} g_{s} \sigma G q\right\rangle}{1024 \pi^{6}} \int \mathrm{d} y \mathrm{~d} z(1-y-z)\left(s-\bar{m}_{c}^{2}\right)^{2} \\
& +\frac{m_{c}\left\langle\bar{q} g_{s} \sigma G q\right\rangle}{65536 \pi^{6}} \int \mathrm{d} y \mathrm{~d} z\left(\frac{1}{y}+\frac{1}{z}\right) \\
& \times(1-y-z)^{2}\left(s-\bar{m}_{c}^{2}\right)^{2} \\
& -\frac{m_{c}\left\langle\bar{q} g_{s} \sigma G q\right\rangle}{98304 \pi^{6}} \int \mathrm{d} y \mathrm{~d} z\left(\frac{1}{y}+\frac{1}{z}\right) \\
& (1-y-z)^{3}\left(s-\bar{m}_{c}^{2}\right)^{2} \\
& +\frac{3 m_{c}\left\langle\bar{q} g_{s} \sigma G q\right\rangle}{16384 \pi^{6}} \int \mathrm{d} y \mathrm{~d} z(1-y-z)\left(s-\bar{m}_{c}^{2}\right)^{2},
\end{aligned}
$$

$$
\begin{aligned}
\rho_{6}^{1}(s)= & \frac{\langle\bar{q} q\rangle^{2}}{96 \pi^{4}} \int \mathrm{d} y \mathrm{~d} z y z(1-y-z)\left(s-\bar{m}_{c}^{2}\right)\left(2 s-\bar{m}_{c}^{2}\right), \\
\widetilde{\rho}_{6}^{0}(s)= & \frac{\langle\bar{q} q\rangle^{2}}{384 \pi^{4}} \int \mathrm{d} y \mathrm{~d} z(y+z)(1-y-z)\left(s-\bar{m}_{c}^{2}\right) \\
& \times\left(3 s-\bar{m}_{c}^{2}\right),
\end{aligned}
$$

$$
\begin{aligned}
\rho_{8}^{1}(s)= & -\frac{35\langle\bar{q} q\rangle\left\langle\bar{q} g_{s} \sigma G q\right\rangle}{6144 \pi^{4}} \int \mathrm{d} y \mathrm{~d} z y z\left(3 s-2 \bar{m}_{c}^{2}\right) \\
& -\frac{\langle\bar{q} q\rangle\left\langle\bar{q} g_{s} \sigma G q\right\rangle}{12288 \pi^{4}} \\
& \times \int \mathrm{d} y \mathrm{~d} z(y+z)(1-y-z)\left(5 s-4 \bar{m}_{c}^{2}\right), \\
\widetilde{\rho}_{8}^{0}(s)= & -\frac{35\langle\bar{q} q\rangle\left\langle\bar{q} g_{s} \sigma G q\right\rangle}{12288 \pi^{4}} \int \mathrm{d} y \mathrm{~d} z(y+z)\left(2 s-\bar{m}_{c}^{2}\right) \\
& -\frac{\langle\bar{q} q\rangle\left\langle\bar{q} g_{s} \sigma G q\right\rangle}{6144 \pi^{4}} \int \mathrm{d} y \mathrm{~d} z \\
& \times(1-y-z)\left(4 s-3 \bar{m}_{c}^{2}\right),
\end{aligned}
$$$$
\rho_{9}^{1}(s)=-\frac{m_{c}\langle\bar{q} q\rangle^{3}}{144 \pi^{2}} \int_{y_{i}}^{y_{f}} \mathrm{~d} y,
$$$$
\widetilde{\rho}_{9}^{0}(s)=-\frac{m_{c}\langle\bar{q} q\rangle^{3}}{72 \pi^{2}} \int_{y_{i}}^{y_{f}} \mathrm{~d} y,
$$$$
\rho_{10}^{1}(s)=\frac{19\left\langle\bar{q} g_{s} \sigma G q\right\rangle^{2}}{24576 \pi^{4}} \int_{y_{i}}^{y_{f}} \mathrm{~d} y y(1-y)
$$$$
\times\left[2+\tilde{m}_{c}^{2} \delta\left(s-\tilde{m}_{c}^{2}\right)\right]
$$$$
+\frac{17\left\langle\bar{q} g_{s} \sigma G q\right\rangle^{2}}{442368 \pi^{4}} \int \mathrm{d} y \mathrm{~d} z(y+z)
$$ 
35. V. Kubarovsky, M.B. Voloshin, Phys. Rev. D 92, 031502 (2015)

36. M. Karliner, J.L. Rosner, Phys. Lett. B 752, 329 (2016)

37. Z.G. Wang, T. Huang, Eur. Phys. J. C 74, 2891 (2014)

38. Z.G. Wang, Eur. Phys. J. C 74, 2963 (2014)

39. Z.G. Wang, Int. J. Mod. Phys. A 30, 1550168 (2015)

40. R.D. Matheus, S. Narison, M. Nielsen, J.M. Richard, Phys. Rev. D 75, 014005 (2007)

41. F.S. Navarra, M. Nielsen, S.H. Lee, Phys. Lett. B 649, 166 (2007)

42. Z.G. Wang, Eur. Phys. J. C 59, 675 (2009)

43. Z.G. Wang, Eur. Phys. J. C 63, 115 (2009)

44. J.R. Zhang, M.Q. Huang, Commun. Theor. Phys. 54, 1075 (2010)

45. M. Nielsen, F.S. Navarra, S.H. Lee, Phys. Rept. 497, 41 (2010)

46. Z.G. Wang, T. Huang, Phys. Rev. D 89, 054019 (2014)

47. Z.G. Wang, Mod. Phys. Lett. A 29, 1450207 (2014)

48. Z.G. Wang, Y.F. Tian, Int. J. Mod. Phys. A 30, 1550004 (2015)

49. A. De Rujula, H. Georgi, S.L. Glashow, Phys. Rev. D 12, 147 (1975)

50. T. DeGrand, R.L. Jaffe, K. Johnson, J.E. Kiskis, Phys. Rev. D 12, 2060 (1975)

51. Z.G. Wang, Eur. Phys. J. C 71, 1524 (2011)

52. R.T. Kleiv, T.G. Steele, A. Zhang, Phys. Rev. D 87, 125018 (2013)

53. Z.G. Wang, Commun. Theor. Phys. 59, 451 (2013)

54. Z.G. Wang, Eur. Phys. J. C 68, 479 (2010)
55. M.A. Shifman, A.I. Vainshtein, V.I. Zakharov, Nucl. Phys. B 147(385), 448 (1979)

56. L.J. Reinders, H. Rubinstein, S. Yazaki, Phys. Rept. 127, 1 (1985)

57. S. Huang, Free particles and fields of high spins (in chinese). (Anhui Peoples Publishing House, Anhui, 2006)

58. K.A. Olive et al., Chin. Phys. C 38, 090001 (2014)

59. Z.G. Wang, Phys. Lett. B 685, 59 (2010)

60. Z.G. Wang, Eur. Phys. J. C 68, 459 (2010)

61. Z.G. Wang, Eur. Phys. J. A 45, 267 (2010)

62. Z.G. Wang, Eur. Phys. J. A 47, 81 (2011)

63. Z.G. Wang, Commun. Theor. Phys. 58, 723 (2012)

64. Z.G. Wang, T. Huang, arXiv:1508.04189

65. Z.G. Wang, arXiv: 1509.06436

66. Z.G. Wang, arXiv: 1512.04763

67. J.M. Dias, F.S. Navarra, M. Nielsen, C.M. Zanetti, Phys. Rev. D 88, 016004 (2013) 